\title{
Design study of 8 meter monolithic mirror UV/optical space telescope
}

\author{
H. Philip Stahl \\ NASA Marshall Space Flight Center, Huntsville, AL 35812
}

\begin{abstract}
The planned Ares V launch vehicle with its 10 meter fairing shroud and 55,000 kg capacity to the Sun Earth L2 point enables entirely new classes of space telescopes. NASA MSFC has conducted a preliminary study that demonstrates the feasibility of launching a 6 to 8 meter class monolithic primary mirror telescope to Sun-Earth L2 using an Ares V. Specific technical areas studied included optical design; structural design/analysis including primary mirror support structure, sun shade and secondary mirror support structure; thermal analysis; launch vehicle performance and trajectory; spacecraft including structure, propulsion, GN\&C, avionics, power systems and reaction wheels; operations \& servicing; mass and power budgets; and system cost.
\end{abstract}

Keywords: Large Space Telescopes, UV/Optical Space Telescopes, Ares V Launch Vehicle, Astronomy

\section{INTRODUCTION}

An 8-meter class space telescope offers the opportunity to answer some of the most compelling science questions. How did the present Universe come into existence and of what is it made? What are the fundamental components that govern the formation of today's galaxies? How does the Solar System work? What are the conditions for planet formation and the emergence of life? And maybe most importantly, are we alone? (Postman, 2008) A recent design study conducted at Marshall Space Flight Center has shown that it is possible to package a 6 to 8 meter class monolithic observatory into a 10 meter Ares V fairing (Figure 1); have it survive launch; and place it in to a halo orbit about the Sun-Earth L2 point.

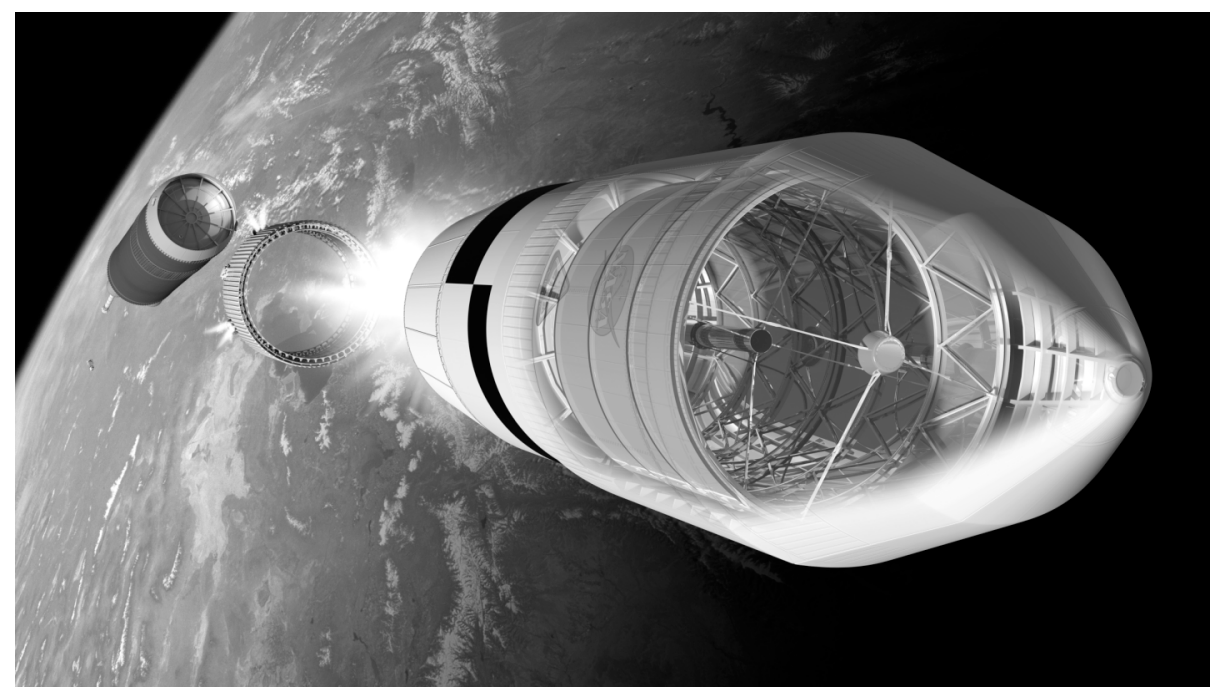

Figure 1 Ares $\mathrm{V}$ can launch 6 to 8 meter class monolithic mirror telescope. (Image courtesy of Jack Frassanito \& Associates and Harley Thronson)

Specific technical areas studied included optical design; structural design/analysis including primary mirror support structure, sun shade and secondary mirror support structure; thermal analysis; launch vehicle performance and trajectory; spacecraft including structure, propulsion, GN\&C, avionics, power systems and reaction wheels; operations \& servicing; mass and power budgets; and system cost. 


\section{THE ARES-V LAUNCH CAPABILITY ENABLES NEW DESIGN CONCEPTS}

The study started with the unique capabilities of the Ares V vehicle (Figure 2) and then considered how those capabilities might enable entirely new mission architectures.

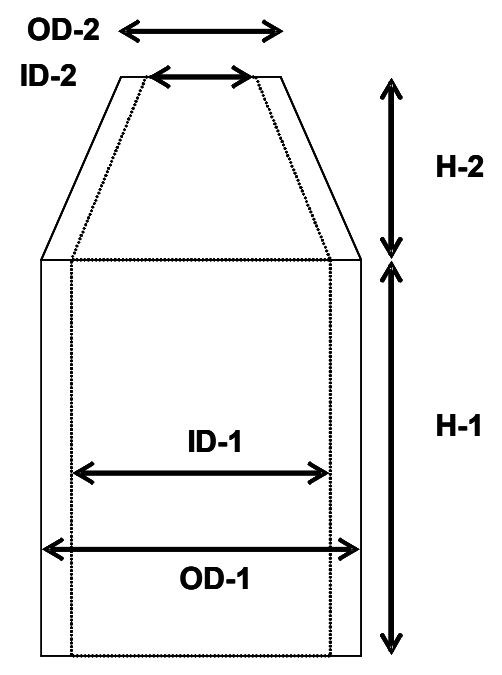

\begin{tabular}{|c|c|c|}
\hline Shroud Outer Diameter & $10-\mathrm{m}$ & \\
\hline Shroud Mass & 7.8 & $\mathbf{m}^{-}$ \\
\hline OD-1 & 10 & $\mathbf{m}$ \\
\hline ID-1 & 8.8 & m \\
\hline$H-1$ & 9.7 & m \\
\hline OD-2 & 5.6 & $\mathbf{m}$ \\
\hline ID-2 & 4.4 & m \\
\hline $\mathrm{H}-2$ & 7.5 & m \\
\hline Total Height & 17.2 & m \\
\hline Volume & 860 & m: \\
\hline Payload to L2TO & 55.8 & $\mathbf{m}$ \\
\hline
\end{tabular}

Figure 2 Ares V Baseline Shroud Dimensions and Payload Mass Capability. (Please note that the Ares V is an evolving vehicle and these are preliminary values and may not match the latest Ares V shroud dimensions and weights.)

First, the baseline 10 meter fairing has an 8.8 meter internal dynamic envelope diameter. This is sufficient to accommodate an 8-meter class monolithic circular primary mirror without the need for segmentation. A monolithic mirror provides superior science return because, as compared to a segmented mirror, it has a more uniform, symmetric and stable Point Spread Function. And, it avoids the risk of deployment and complex alignment and phasing control. The 10 meter shroud also allows an 8-meter monolithic mirror to be launched in a face up configuration which provides the most benign vibration and acoustic exposure. Looking further into the future, the 10 meter fairing also allows for even larger aperture segmented designs. Concepts are under consideration for 16 to 24 meter segmented telescopes.

Second, the payload mass of 55,800 $\mathrm{kg}$ to an L2 Transfer Orbit enables an entirely new paradigm - design simplicity. Given the available of extra mass, use more mature technologies and higher design rule safety factors to eliminate complexity, to lower cost and to lower risk. By using higher design margins it is possible to minimize the marching army size which also reduces the management burden - every $\$ 100 \mathrm{M}$ in component cost savings reduces total program cost from $\$ 300 \mathrm{M}$ to $\$ 500 \mathrm{M}$. The cost savings of eliminating mass constraint is difficult to quantify, but anecdotal evidence suggests that early in a mass constrained mission, it may cost $\$ 100 \mathrm{~K}$ of design effort to eliminate $1 \mathrm{~kg}$ of mass and that once the design is mature, it can cost as much as $\$ 1 \mathrm{M}$ to eliminate $1 \mathrm{~kg}$ of mass.

These two unprecedented enabling capabilities of the Ares $\mathrm{V}$ formed the basis for the foundational question of the MSFC design study. Is it possible to launch an 8-meter class space observatory using a conventional massive monolithic ground based telescope mirror? Instead of using lightweight (very expensive and high risk) mirror technology, it is possible to use a conventional massive (low cost and low risk) ground telescope mirror? And the answer is YES. 


\section{OBSERVATORY DESIGN}

\subsection{Design Concept}

Figure 3 shows the MSFC design concept for an 8-meter monolithic primary mirror ultraviolet/optical space observatory packaged inside the Ares V 10-m fairing's dynamic envelope. The concept has three main subsystems: telescope, support structure and spacecraft. The telescope consists of an 8-meter primary mirror, secondary mirror and forward structure/baffle tube. The spacecraft provides all normal spacecraft functions (such as propulsion; guidance, navigation and control; communication; etc.) and houses the science instruments. The support structure supports the primary mirror. And, it carries the observatory mass (of the primary mirror, telescope forward structure and spacecraft) providing the interface of this mass to the Ares $V$ for launch.

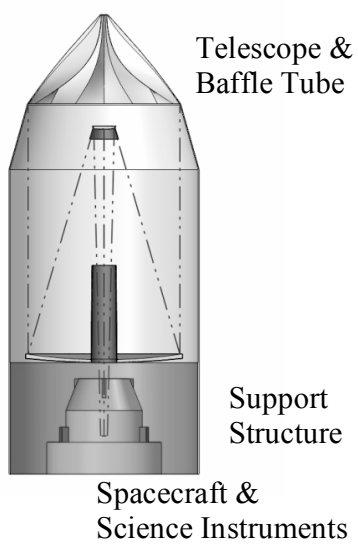

Figure 3 MSFC 8-meter observatory concept in Ares V dynamic envelope

\subsection{Optical Design}

The feasibility study considered two different telescope optical systems. An F/15 Ritchey-Chretién design (Figure 4a) was examined for its excellent on- and off-axis image quality, compact size, and ultra-violet throughput. Also RC designs are the optical system mainly used by today's large telescopes. Therefore, it might be possible to reuse existing scientific instrument designs. Unfortunately, this optical design has only a relatively narrow 1 -arc minute field of view (NFOV) that is diffraction limited at $500 \mathrm{~nm}$. One method to achieve the desired wide field performance is to use a refractive corrector - although with a limited spectral range - in the scientific instrument suite. Another approach to achieving multi-spectral wide field performance is to use a three mirror anastigmatic telescope with fine steering mirror design (Figure 4b). This configuration has a wide 100 arc-minute ( 8.4 by 12 arc minutes) field of view (WFOV) that is diffraction limit at $500 \mathrm{~nm}$. But, it also has lower ultra-violet throughput because of its two additional reflections. A potential solution is to implement a dual pupil configuration where UV and NFOV instruments operate at the Cassegrain focus and WFOV instruments operate off-axis providing their own tertiary mirror.
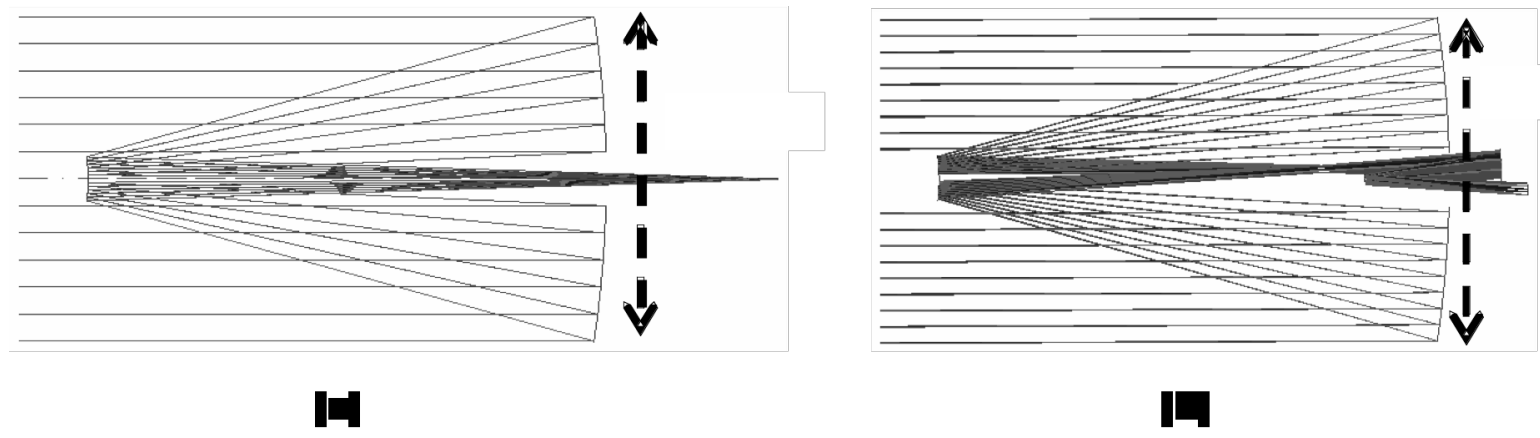

Figure 4 Telescope Optical Path. The original optical configuration (a) was used to size the telescope subsystems and develop the mass budget. The revised optical configuration (b) results in an $80 \mathrm{X}$ larger field of view

\subsection{Primary Mirror}

For either telescope design, the monolithic mirror will be manufactured using existing ground based mirror technology. This approach has two specific advantages: technical maturity and cost risk. First, it has been demonstrated that one can actually polish an 8 meter class ground based telescope mirror to a surface figure of better than $8 \mathrm{~nm}$ rms (Geyl, 1999) (which is close to the desired $5 \mathrm{~nm}$ rms surface figure for the 8 meter Terrestrial Planet Finder program). This is important because as shown in Table 1, while Hubble's 2.4 meter $180 \mathrm{~kg} / \mathrm{m} 2$ mirror was polished to $6.4 \mathrm{~nm} \mathrm{rms}$, the AMSD program only achieved $20 \mathrm{~nm}$ rms on its 1.4 meter segment $18 \mathrm{~kg} / \mathrm{m} 2$ mirror. The higher the mirror's areal density, the easier it is to achieve a very good surface figure. Second, the cost for an 8 meter ground mirror is approximately $\$ 20 \mathrm{M}$ or $\$ 0.4 \mathrm{M} / \mathrm{m} 2$ while the cost of a 50 square meter space technology mirror will be $\$ 200$ to $\$ 500 \mathrm{M}$ $(\$ 4 \mathrm{M}$ to $\$ 10 \mathrm{M} / \mathrm{m} 2)$. While this architectural choice adds approx $20,000 \mathrm{~kg}$ to the mass of the payload, the estimated 
$\$ 200 \mathrm{M}$ to $\$ 500 \mathrm{M}$ savings in mirror hardware costs translates into total program cost savings of from $\$ 700 \mathrm{M}$ to $\$ 2 \mathrm{~B}$ (engineering design, system integration \& test, management and fees/program reserves add to the total cost of any program by a factor of $2.5 \mathrm{X}$ to $3 \mathrm{X}$ of the hardware costs).

\begin{tabular}{|l|c|c|c|c|c|l|}
\hline \multicolumn{7}{|c|}{ Table 1. Comparison of Space and Ground Mirrors } \\
\hline Parameter & Spitzer & HST & AMSD & JWST & Ground & \\
\hline Material & Beryllium & ULE & ULE \& Be & Be & Various Glass & \\
\hline Diameter & 0.85 & 2.4 & 1.4 & $1.5(6.5)$ & 8.2 & $\mathrm{~m}$ \\
\hline Area & 0.5 & 4.5 & $\sim 1$ & 25 & 50 & $\mathrm{~m} 2$ \\
\hline Temperature & 4 & 300 & $300 / 30$ & 30 & 300 & $\mathrm{~K}$ \\
\hline Surface Figure & 75 & 6.4 & $20 / 77$ & 25 & 8 & $\mathrm{~nm} \mathrm{rms}$ \\
\hline Areal Density & 28 & 180 & 18 & 26 & 460 & $\mathrm{~kg} / \mathrm{m} 2$ \\
\hline Areal Cost & 10 & 10 & 4 & 6 & 0.5 & $\$ \mathrm{M} / \mathrm{m} 2$ \\
\hline Year & $199 ?$ & $198 ?$ & 2005 & 2008 & 2008 & \\
\hline
\end{tabular}

The reason for both advantages is that ground based mirrors are very massive and hence very stiff. Thus, they are much easier to fabricate than space mirrors. Historically, space mirrors are very low mass (Table 1) and thus not very stiff. They have large gravity sags and are difficult to handle, mount and fixture. And, they are difficult to fabricate to very high precision. Thus, they are expensive.

Three ground based mirror technologies have been considered. The spare VLT (Very Large Telescope) mirror manufactured by Schott is an 8.2 meter diameter, $200 \mathrm{~mm}$ thick, Zerodur solid thin meniscus blank with a mass of $23,000 \mathrm{~kg}$. If edged to 6.2 meters diameter and $175 \mathrm{~mm}$ thick, it would have a mass of $11,000 \mathrm{~kg}$. The University of Arizona manufactures 6 to 8 meter class borosilicate mirrors using their honeycomb spin cast technique. Recently, Arizona manufacture two mirrors for the LBT (Large Binocular Telescope) that are 8.4 meter diameter, $900 \mathrm{~mm}$ thick and with a mass of $16,000 \mathrm{~kg}$. Finally, Corning manufactured the Subaru Telescope ULE thin meniscus 8.3 meter primary mirror blank with a mass of $21,000 \mathrm{~kg}$.

\subsection{Structural Design}

A fundamental question of the design study was whether an 8-meter class ground based telescope mirror could even survive launch. The Ares $\mathrm{V}$ launch environment was analyzed by the MSFC Advanced Concepts Office using POST3D. The maximum launch loads (Table 2) are similar to those for existing launch vehicles. Please note, these loads are not concurrent. They are the maximum load experienced at some time during launch. And, the lateral loads do not include wind loading or vibration.

\begin{tabular}{|l|c|}
\hline \multicolumn{2}{|c|}{$\begin{array}{c}\text { Table } 2 \text { Maximum Launch Loads of an Ares V } \\
\text { via POST3D Analysis }\end{array}$} \\
\hline \multicolumn{1}{|c|}{ Maximum Launch Load } & Ares V \\
\hline Axial (Z) & 4 g's \\
\hline Lateral (Y) & 7 x 10-6 g's \\
\hline Lateral (Z) (Z is down range direction) & $6 \times 10-4$ g's \\
\hline
\end{tabular}

A structural analysis determine that 66 axial support points keeps the stress level on an 8.2 meter diameter $175 \mathrm{~mm}$ thick meniscus primary mirror below 1000 psi (Figure 5). Thus, the mirror will survive launch.
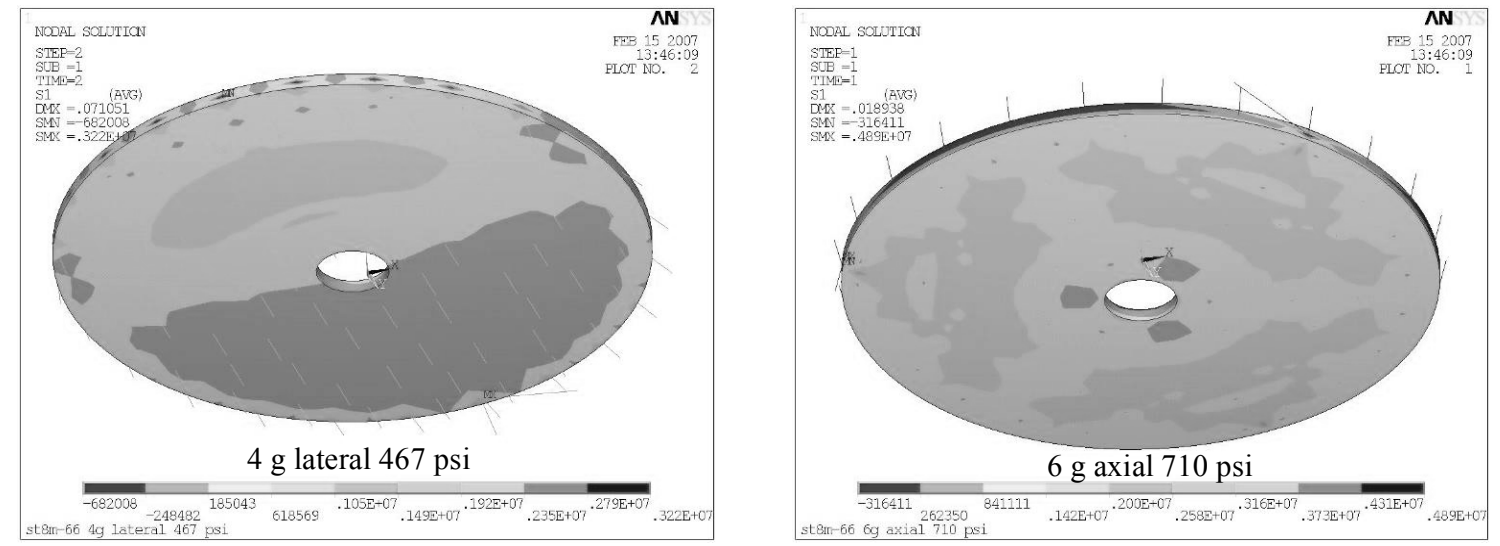

Figure 5 An 8.2 meter 175 mm thick mirror can survive launch loads. 66 axial supports keep bulk stress below 1000 psi. 
The observatory structure is divided between the forward and back structure. The forward structure is similar to that of the Hubble Space Telescope. It provides the metering structure between the primary and secondary mirrors and holds the straylight baffle tube. Because of fairing length limitations, the forward structure is split into an upper and lower part. The lower structure is load carrying. It holds the secondary mirror assembly tripod structure. The upper part contains the upper baffle tube and the cover doors. The upper part slides forward on orbit to the full length of the straylight baffle. The cover doors open and close on-orbit as required.

The back structure has multiple functions. First, it supports the primary mirror with 66 axial supports. Second, the forward structure is attached to the back structure as is the spacecraft. A key design element of the MSFC Structural concept is that all the mass of the observatory is carried through the main support structure to an interface ring which attaches via another support system to the Ares $\mathrm{V}$ launch vehicle. This design concept allows the use of a completely conventional spacecraft, i.e. it does not need extra mass because it does not provide the interface between the observatory and the launch vehicle.

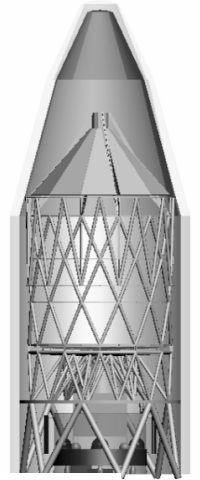

Figure 6 Observatory Support Structure

Structural design and analysis was performed for the spacecraft using standard NASA guidelines. No technical problems were identified. The primary product of this effort was a mass budget for the spacecraft

\subsection{Thermal Design}

Standard thermal design and analysis was performed for 4 different solar angles: $0,45,90$ and 120 degrees where 0 degrees is the observatory back facing the sun and 90 degrees is the observatory broadside to the sun. It was modeled that the science instruments produce $750 \mathrm{~W}$ of heat and the avionic systems produce another $850 \mathrm{~W}$ of heat. The analysis assumed that the observatory is wrapped with five 10 layer MLI blankets and that the spacecraft has $16.0 \mathrm{~m} 2$ of thermal radiators. Thermal gradients were calculated for both the spacecraft and the 8 meter primary mirror. (Figure 7)

Without an active thermal management system, the primary mirror temperature varies as a function of sun angle from $160 \mathrm{~K}$ to $300 \mathrm{~K}$ with approximately a $1 \mathrm{~K}$ variation at each temperature.

\begin{tabular}{|c|c|}
\hline Table 3 Primary Mirror Temperature \\
\hline Sun Angle & Temperature \\
\hline $0 \mathrm{deg}$ & $200 \mathrm{~K}$ \\
\hline $45 \mathrm{deg}$ & $190 \mathrm{~K}$ \\
\hline $90 \mathrm{deg}$ & $160 \mathrm{~K}$ \\
\hline $120 \mathrm{deg}$ & $300 \mathrm{~K}$ \\
\hline
\end{tabular}
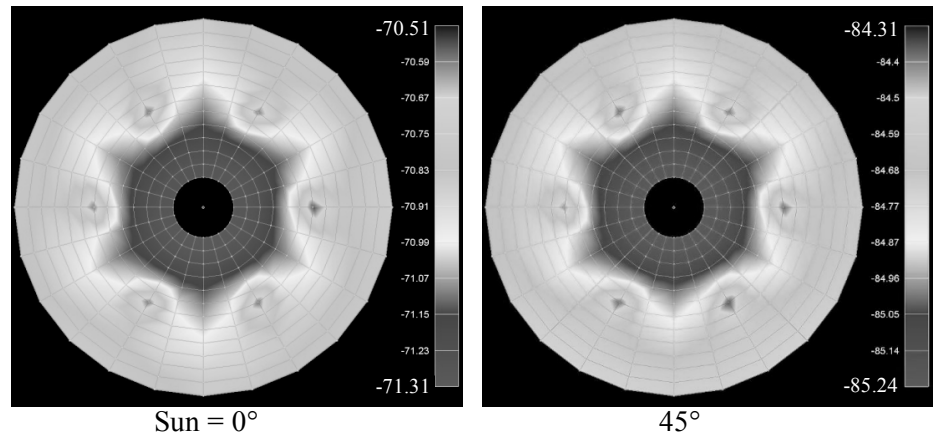

Therefore, an active thermal management via 14 heat pipes is required to hold the primary mirror temperature at a constant $300 \mathrm{~K}$ for all sun angles with less than $1 \mathrm{~K}$ of thermal gradient. On-going thermal analysis will determine exactly how small of a thermal gradient can be achieved. This is important for long exposure observations which required a very stable observatory wavefront (such as extra-solar terrestrial planet finding and characterization). The primary mirror surface figure varies as a function of temperature based on the substrate material CTE value and uniformity.

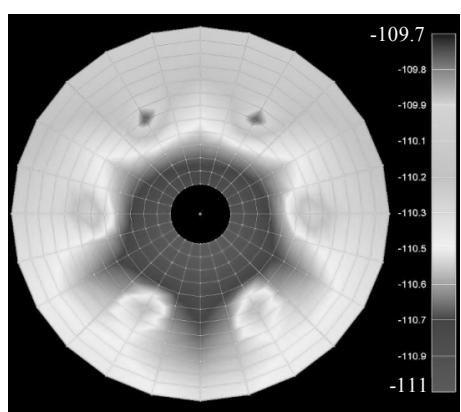

$90^{\circ}$

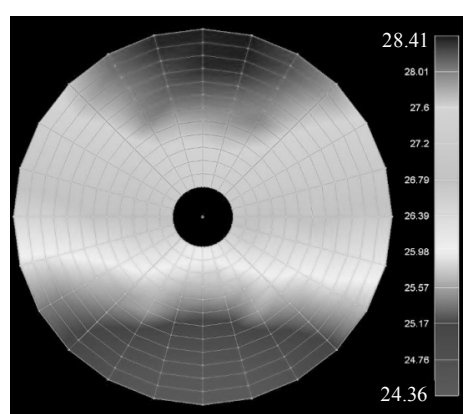

$120^{\circ}$

Figure 7 Primary mirror temperature $\left({ }^{\circ} \mathrm{C}\right)$ vs sun angle At $300 \mathrm{~K}$, Corning specifies ULE to have a mean CTE value of $0+/-30 \mathrm{ppb} / \mathrm{K}$ from $5 \mathrm{C}$ to $35 \mathrm{C}$ and Schott specifies that Zerodur has a CTE value of $0+/-50 \mathrm{ppb} / \mathrm{K}$ with a uniformity of $+/-10 \mathrm{ppb} / \mathrm{K}$. 


\subsection{Spacecraft}

The observatory has two separate spacecraft: a telescope bus which houses the optical telescope element (OTE), and a replaceable spacecraft/instrument bus (SIB) (Figure 8). The SIB houses science instruments and subsystems to communicate with and control the telescope. Each spacecraft produces its own power. The telescope has $18 \mathrm{~m} 2$ of body mounted solar arrays around the light tube. The SIB has $9 \mathrm{~m} 2$ of deployable solar array wings with pointing ability. The SIB power system includes $800 \mathrm{~W}$ for primary mirror thermal control and $750 \mathrm{~W}$ for science instruments. The OTE performs its own on-board health diagnostics and communication to the SIB. The SIB provides the primary communication down-link.

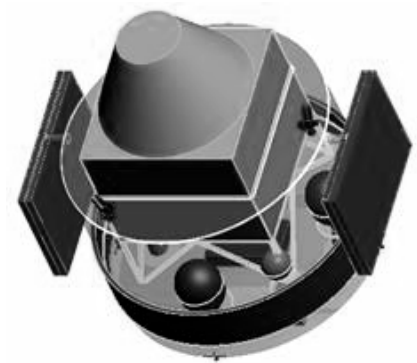

Figure 8 Spacecraft/Instrument Bus (SIB). Top contains science instruments, Solar panels are on side.

The spacecraft propulsion system is sized to get the observatory from roughly a geostationary transfer orbit (energy, or $\mathrm{C} 3$, of $-2.60 \mathrm{~km}^{2} / \mathrm{s}^{2}$ ) into a halo orbit about the Sun-Earth L2 point and performs all station keeping operations. The spacecraft has a dual mode hydrazine-NTP bi-prop / hydrazine mono-prop propulsion system with 5 years of propellant and redundant thrusters. The propellant load is based on an estimated V expenditure for five years of $20 \mathrm{~m} / \mathrm{s}$. Propulsion during the trip from GTO to L2 is provided by hydrazine-BTP bi-prop $125 \mathrm{lbf}$ thrusters (Northrop). Station keeping at L2 is provided by hydrazine mono-prop RCS 20/5 lbf thrusters (Aerojet). The telescope has an independent control system with mono-propellant hydrazine using 350/100 psi blowdown Aerojet thrusters. The telescope propulsion system has $30 \mathrm{~kg}$ of propellant for 30 year mission.

Guidance Navigation and Point Control is provided by the spacecraft reaction wheels. A trade study was performed to obtain the optimum science performance as a function of wheel torque and momentum storage specifications. (Figure 9) Two performance parameters were analyzed. The number of hours that the telescope can stare at a fixed point in space (remain at an inertial hold) before needing to perform a momentum dump due to solar radiation pressure torque. And, how fast in minutes the telescope can perform a 60 degree slew. The analysis was done for a sun angle of 90 degrees with is the worst condition for solar radiation pressure torque. At any other sun angle, the available science time increases. And it was assumed that momentum buildup occurs in only one axis (y-axis). 6 wheel and 4 wheel configurations were analyzed along with the worst case single wheel failure for each configuration. Each configuration was analyzed for three different TELDIX reaction wheel versions (Torque-Momentum Storage).

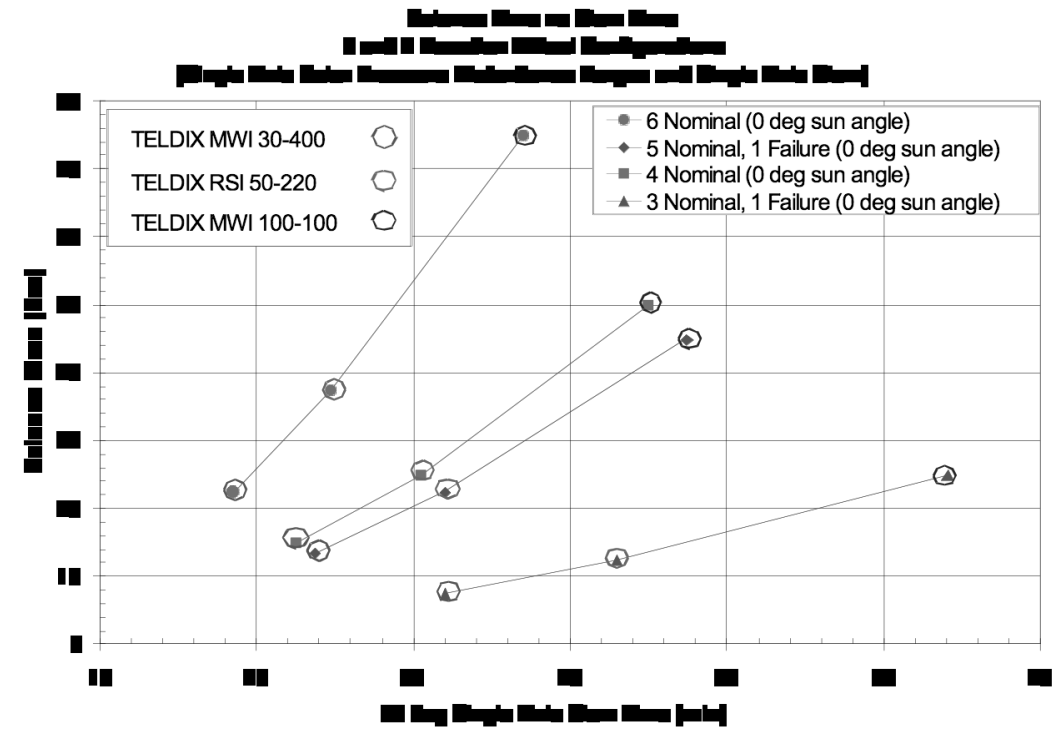

Figure 9 Science Time vs Slew Time Analysis for various Reaction Wheels

\subsection{Mass Budget}

The entire mass budget for the 6-meter observatory including primary mirror, structure, light baffle tube, instruments, space craft, avionics, etc. is less than 35,000 kg (Table 4) - a 38\% mass margin on the Ares V's 55,600 kg Sun-Earth L2 launch capability. The mass budget for an 8 meter observatory is approximately $45,000 \mathrm{~kg}$, with almost a $20 \%$ mass margin, of which the primary mirror is the largest contributor. These mass budgets clearly show that payload diameter/volume, and not the payload mass, is the limiting factor in the telescope size. 
Table 4 Mass Budget for a 6 meter Telescope OTE and Spacecraft/Instrument Bus

Total mass $=$ OTE W / Bus + Spacecraft and Science Inst

OTE W / Bus mass

Primary mirror assembly

Secondary mirror assembly

Telescope enclosure

Avionics Subsystems

Power Subsystems

Thermal Management System

Structures

Propulsion

Propellant

Docking station

Spacecraft and Science Instrument

Science Instrument Package

Avionics Subsystems
Power Subsystems
Thermal Management System
Structures
Propulsion
Propellant
Docking station

Mass $(\mathrm{Kg})$

33,849

25,619

17750

671

3,600

153

381

1,091

917

16

40

1,000

6,230

1500

334

377

481

755

248

1,536

1,000

2,000

Launch Adapter

Please note that several elements of this mass budget are allocations, including the science instrument package, launch adapter and docking stations. All mass elements will be subject to refinement as the design matures.

\section{EXTENDED MISSION LIFE BY IN-SPACE SERVICING}

To extend the life of the observatory beyond its initial design life of 5 years to a target life of 30 years or more, the science instruments and as many subsystem components as possible are designed to be replaced at periodic intervals. These are all in the SIB (Figure 8) which can be replaced as a single unit every 3 to 5 years using autonomous rendezvous and docking (AR\&D) technology (as demonstrated on Orbital Express). Beyond the obvious technical advantages of upgrading detectors, electronics and computers periodically, it is anticipated that designing subsystems for 5 years of operation instead of 10 years will produce sufficient cost savings to fund the periodic servicing missions. (This needs to be the subject of a design trade.) The SIB diameter is set at 4.5 meters such that these servicing missions can be launched via a conventional EELV.

Using this approach, the first 5 years of mission life could be dedicated to UV science with a narrow FOV UV spectrometer and a WFOV UV imager. Then, the next 5 years of mission life could be dedicated to visible science such a terrestrial planet finding with either an external occulter or an internal coronagraph. Eventually, it might be possible to have two different SIBs on station with the ability to switch between suites of science instruments. At the observatory end of life, as thermal control degrades, the telescope can be allowed to cool to $<200 \mathrm{~K}$ for an infrared science campaign.

During the period of SIB exchange, when the SIB is undocked from the observatory, the telescope spacecraft provides basic guidance and navigation for station keeping. The telescope has $18 \mathrm{~m} 2$ of body mounted solar array around light tube, used for station keeping, and batteries for up to 0.5 hour of attitude control contingency. The telescope avionics systems are 3-fault tolerant for a 30 year life. As previously discussed, the telescope has a mono-propellant blow-down thrust system. The telescope also has a low gain antenna for communicating with the servicing spacecraft. All telescope health and status data is sent directly to the spacecraft avionics system. Also, power for the telescope thermal management system is provided by the SIB. Thus, there is no active thermal control during spacecraft exchange. 
The primary subsystems for pointing, communications, power, guidance, propulsion, as well as the science instrument package and fine guidance sensor, are located on the SIB. One notable exception is the thermal control for the primary mirror, which must be placed on the telescope bus. The SIB avionics and power systems are 1-fault tolerant for 5 year life. Power is generated from two $9 \mathrm{~m} 2$ deployable solar array wings with pointing ability. Batteries are sized for 2 hours of power during midcourse and rendezvous operations (when the power arrays are retracted). The SIB power system includes $800 \mathrm{~W}$ for mirror thermal control and $750 \mathrm{~W}$ for the telescope instrument package. The guidance and navigation system includes star trackers, sun sensors and inertial measurement units. AR\&D will be facilitated with a LIDAR long range system and an optical short range system. Computers handle all normal station keeping, maneuvers, data management, and ground communications. And, the communication systems consist of Ka-band HGA for ground, and s-band for local communication and backup capability

\section{COST DISCUSSION}

The proposed 8 meter monolithic telescope concept seeks to disprove the old adage that the primary predictor of mission cost is mass. It is the author's assertion that the Ares V payload mass capability is a disruptive technology that creates a new paradigm - by trading mass for simplicity it is possible to build a telescope with lower cost and lower risk. By eliminating complexity, it should be possible to design and build an 8-meter monolithic telescope with $2 \mathrm{X}$ the collecting area of the 6.5 meter JWST for less cost. Consider for example the complexity difference between packaging a 6.5 meter segmented primary mirror into a 4.5 meter dynamic launch envelope as compared to the simplicity of packaging an 8 meter monolithic mirror into an 8.8 meter dynamic launch envelope. The current cost for the JWST telescope and spacecraft (excluding science instruments and operation) is approximately $\$ 3 \mathrm{~B}$. The total cost for an 8-meter observatory (excluding science instruments and operations is estimated to be $\$ 1 \mathrm{~B}$ to $\$ 1.5 \mathrm{~B}$. To illustrate this point further, consider the telescope primary mirror and its support structure.

Because of launch vehicle payload mass constraints, all previous space based telescopes have required low areal density primary mirrors - the bigger the telescope, the lower its required areal density. For example, the Hubble primary mirror has an areal density of $180 \mathrm{~kg} / \mathrm{m} 2$ for a total mass of $810 \mathrm{~kg}$ or $7.4 \%$ of Hubble's total mass of $11,000 \mathrm{~kg}$. By comparison, the JWST primary mirror has an areal density of $25 \mathrm{~kg} / \mathrm{m} 2$ for a total mass of $625 \mathrm{~kg}$ or $9.6 \%$ of the total JWST observatory mass of $6,500 \mathrm{~kg}$. The explanation is simple, a primary mirror mass is limited to about $10 \%$ of a space observatory and the total mass of the observatory is limited by the launch vehicle. And, because space mirrors have low areal densities, they are difficult to manufacture and thus expensive. Space mirrors are inherently less stiff than ground mirrors. Thus, they have larger gravity sags; exhibit a fabrication effect called quilting; and are difficult to handle, mount and fixture. Because of this mass and stiffness difference, the cost of a space telescope mirror is typically 10X higher than for a ground telescope mirror (Table 1).

The Ares V eliminates this constraint. The 8-meter monolithic concept proposes to use existing ground based mirror technology rather than the ultra light-weight mirror technology required for a large space telescope via an EELV. While this architectural choice adds approx $20,000 \mathrm{~kg}$ to the mass of the payload, it is estimated to save $\$ 200 \mathrm{M}$ to $\$ 500 \mathrm{M}$ in mirror hardware costs and $\$ 700 \mathrm{M}$ to $\$ 2 \mathrm{~B}$ in total program costs. The precursor JWST mirror technology development program AMSD demonstrated that 1.4 meter light-weight beryllium and glass mirrors both cost approx $\$ 4 \mathrm{M}$ per square meter. Currently, the total cost for the 6.5 meter JWST primary mirror is in excess of $\$ 140 \mathrm{M}$ or close to $\$ 6 \mathrm{M} / \mathrm{m} 2$. Thus, a 50 square meter mirror will cost $\$ 200 \mathrm{M}$ to $\$ 300 \mathrm{M}$. Furthermore, It is likely that a UV/Visible quality version of the JWST primary mirror would be even more expensive, maybe $\$ 500 \mathrm{M}$ (using the HST $\$ 10 \mathrm{M} / \mathrm{m} 2$ areal cost). By comparison, 8-meter class (50 square meter) UV/Visible quality ground based telescope mirrors typically cost less than $\$ 20 \mathrm{M}$ or $\$ 0.4 \mathrm{M} / \mathrm{m} 2$. Given that engineering design, system integration \& test, management and fees/program reserves add to the total cost of any program by a factor of $2.5 \mathrm{X}$ to $3 \mathrm{X}$ of the hardware costs, a $\$ 200 \mathrm{M}$ savings in the cost of a primary mirror translates into a $\$ 700 \mathrm{M}$ to $\$ 800 \mathrm{M}$ total program cost savings.

Risk is also significantly lower for a ground based mirror simply because they have been demonstrated. Currently there are nine 8-meter class monolithic telescopes in operation. Some of these mirrors have a surface figure better than $10 \mathrm{~nm}$ rms - close to the requirement for the Terrestrial Planet Finder primary mirror.

Similar cost savings and risk reductions are anticipated for the telescope structure (ATK private conversation). On JWST, the cost of the telescope structure is approximately $2 / 3^{\text {rd }}$ engineering labor and $1 / 3^{\text {rd }}$ fabrication $\&$ tooling. The 
primary driver for the engineering labor is the need to design a very low mass structure at the limit of performance safety factors. It has been estimated that if the mass could be increased by several factors ( $3 \mathrm{X}$ to $5 \mathrm{X})$ that the engineering time could be cut substantially. For an 8 meter class structure with a mass allocation of 10,000 kg, it is estimated that engineering labor will account for $1 / 3^{\text {rd }}$ of the total cost with the balance for fabrication. And, that the total cost for an 8 meter telescope should not exceed the total cost of JWST (note also that JWST is cryogenic and the proposed UV/optical telescope operates at ambient temperature).

\section{CONCLUSION}

The unprecedented mass and volume capabilities of NASA's planned Ares V cargo launch vehicle enable entire new mission concepts. Its 10 meter fairing and ability to place 55,600 kg of payload into Sun-Earth L2 completely changes the paradigm for future space telescopes - simplicity. Simple high TRL technology offers lower cost and risk. The Ares $\mathrm{V}$ capacities allow one to use mass to buy down performance, cost and schedule risk. Instead of expensive lightweight space mirrors, one can use low-cost low-risk proven ground based mirror technology. And, instead of expending excessive amounts of engineering design and analysis labor, the Ares $\mathrm{V}$ payload capacity allows for mission designs with larger than normal structural safety margins and fewer complex deployment mechanisms.

NASA Marshall Space Flight Center has conducted a preliminary design study which indicates that it is feasible to launch a 6 to 8 meter class monolithic primary mirror ultraviolet/visible observatory. An 8-meter class UV/optical space observatory with its very high angular resolution, very high sensitivity, broad spectral coverage, and high performance stability offers the opportunity to answer some of the most compelling science questions. How did the present Universe come into existence and of what is it made? What are the fundamental components that govern the formation of today's galaxies? How does the Solar System work? What are the conditions for planet formation and the emergence of life?

And maybe most importantly, are we alone?

Finally, there is no inherent reason that an 8-meter space telescope using robust design concepts should have only a 5 to 10 year mission life. In fact, there is no reason that the telescope might not last 20 to 30 years. This extended mission life can be obtained via periodic robotic servicing of the spacecraft and science instruments using autonomous rendezvous and docking technology (as demonstrated on Orbital Express).

\section{ACKNOWLEDGEMENTS}

The author would like to thank Randall Hopkins and Alan Philips of the Marshall Space Flight Center Advanced Concepts Office who provided information, analysis, data and valuable guidance regarding the Ares $\mathrm{V}$ capabilities and preliminary shroud designs and John Hraba, Bill Arnold and Ken Pitalo of the MSFC Optics Office.

\section{REFERENCES}

Postman, Marc, et. al., "Science with an 8-meter to 16-meter optical/UV space telescope", SPIE Proc.7010, (2008). See also white paper submitted to this RFI.

Geyl, R and M. Cayrel. "REOSC Contribution to VLT and Gemini" Part of the EUROPTO Conference on Optical Fabrication and Testing. Berlin, Germany. May 1999. SPIE, Vol. 3739. 1999. 


\section{Design study of an 8-meter monolithic mirror UVIoptical space telescope}

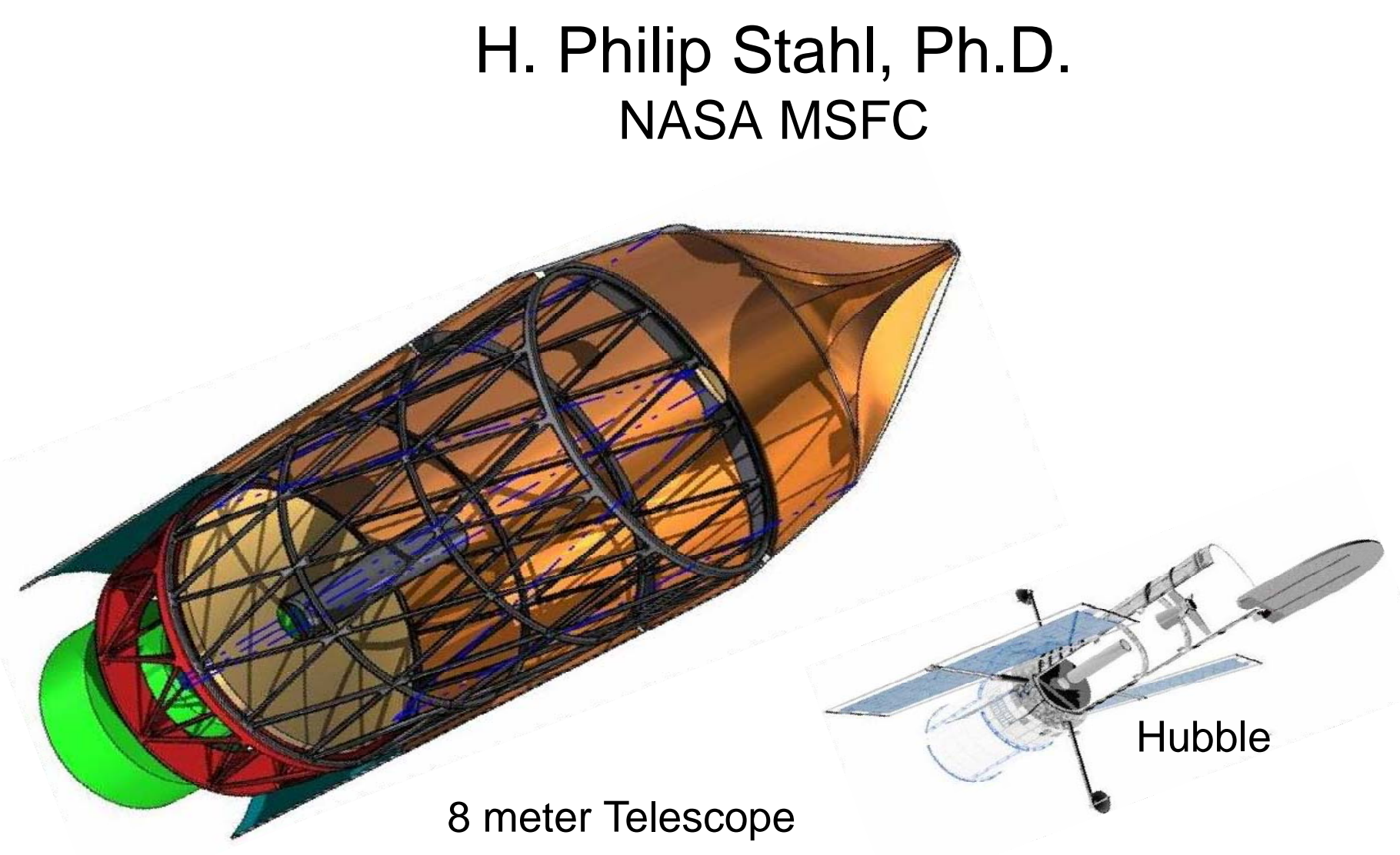




\section{Executive Summary}

The unprecedented volume capability of an Ares $V$ enables the launch of 8 meter class monolithic space telescopes to the Earth-Sun L2 point.

The unprecedented mass capability of an Ares $V$ enables an entirely new design paradigm - Simplicity.

Simple high TRL technology offers lower cost and risk.

NASA MSFC has determined that a 6 to 8 meter class telescope using a massive high-TRL ground observatory class monolithic primary mirror is feasible. 


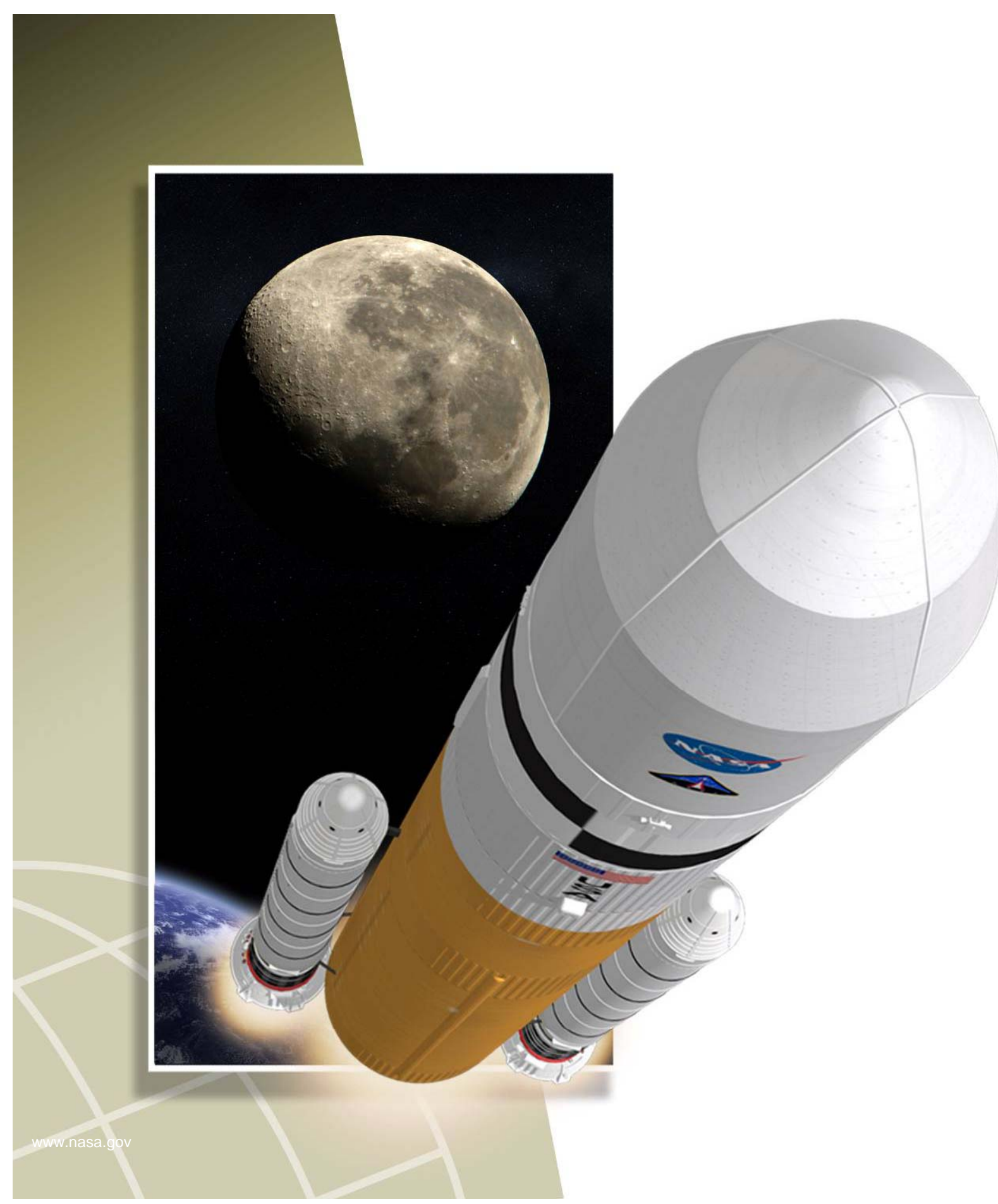

\section{Ares V Performance Capability}

(at this point in time) 
NASA Ares $V$ delivers $5 X$ more Mass to Orbit .

- Sun

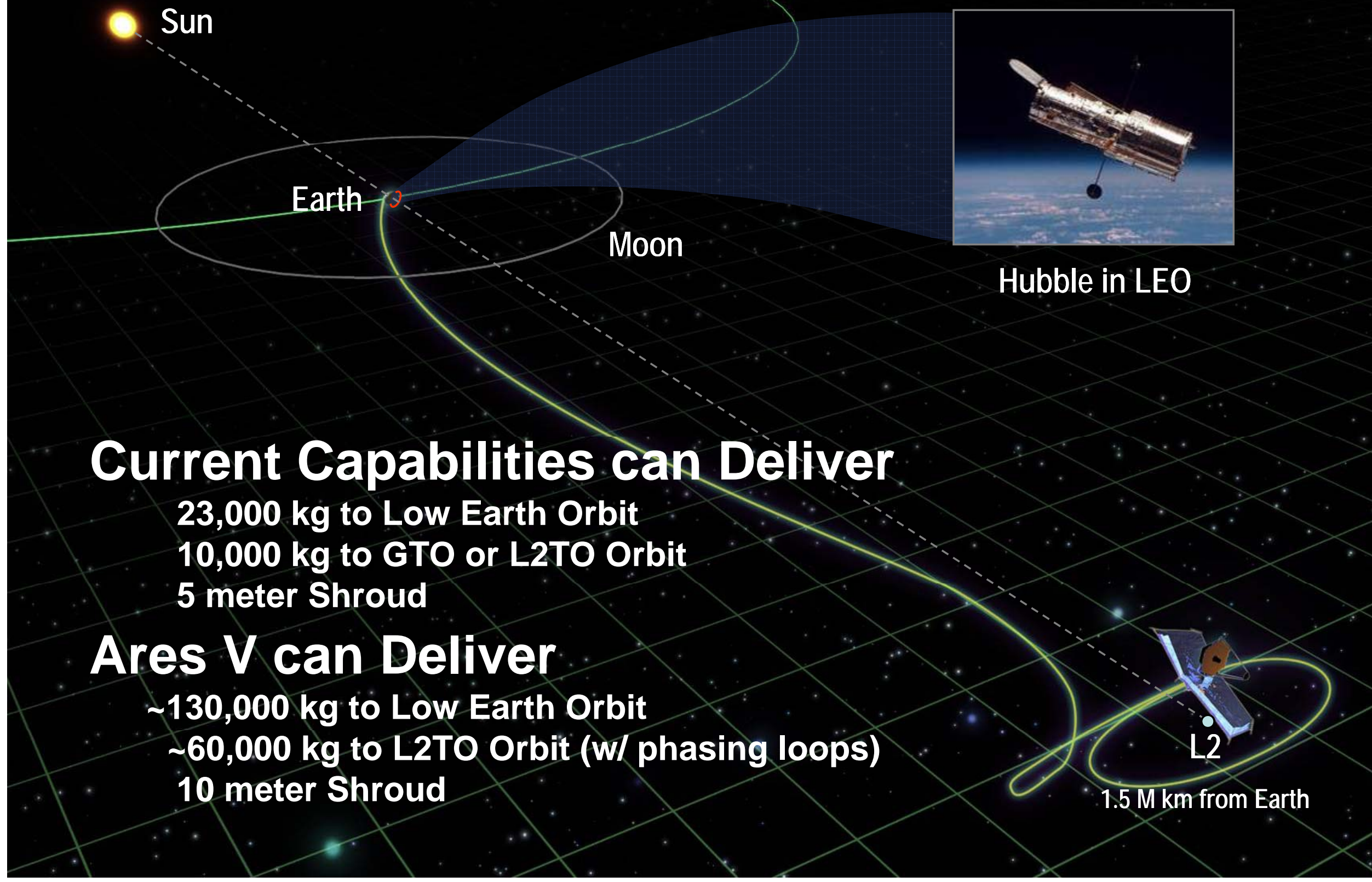




\section{Building on a Foundation of Proven Technologies}

\section{- Launch Vehicle Comparisons -}

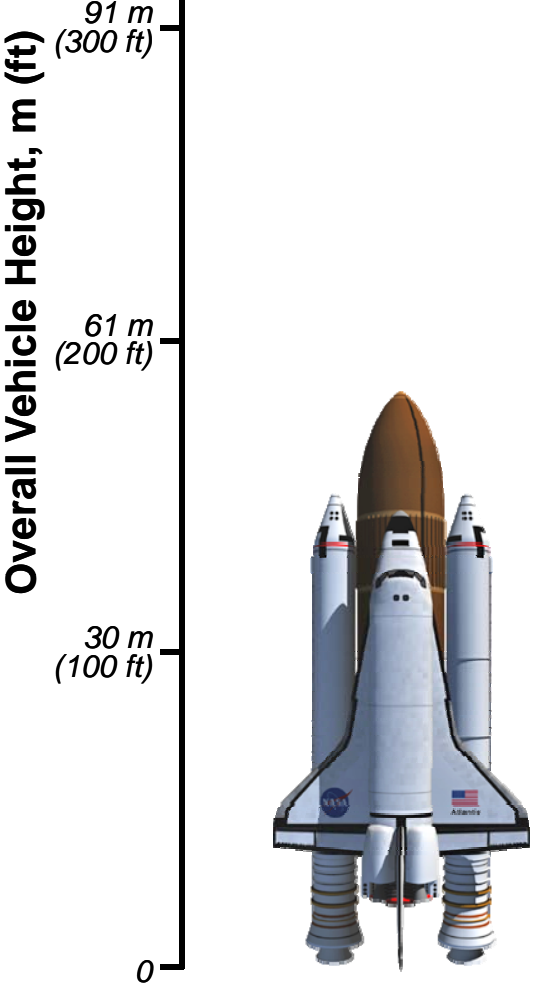

Space Shuttle

Height: $56.1 \mathrm{~m}$ (184.2 ft)

Gross Liftoff Mass:

2,041,166 kg (4.5M lbm)

25 MT (55k lbm)

to Low Earth Orbit (LEO)

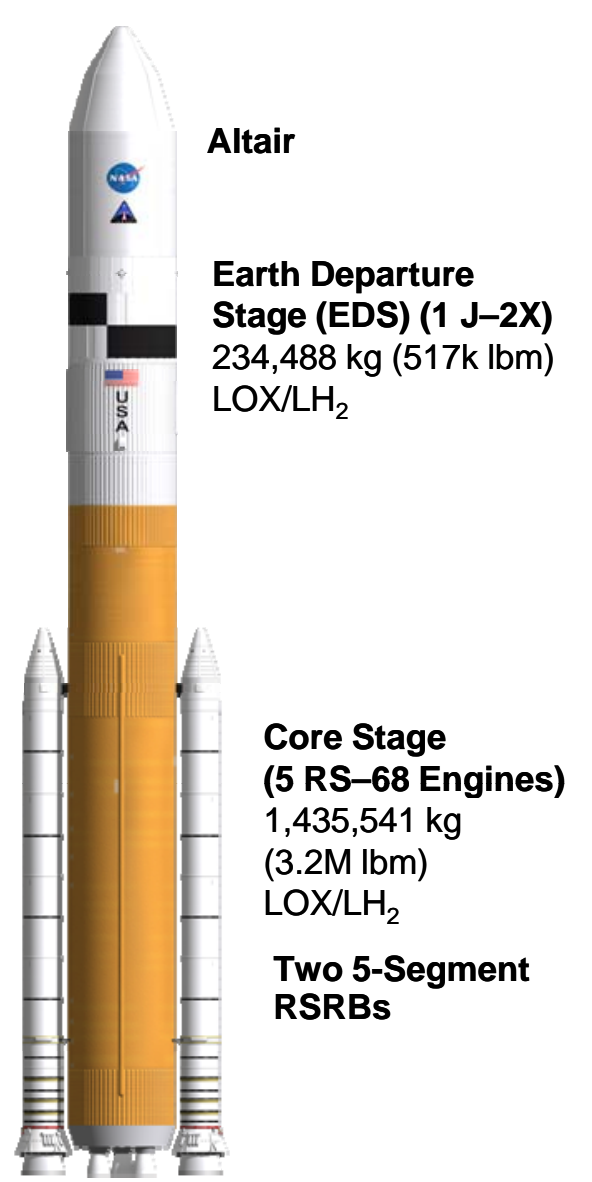

Ares I

Height: $99.1 \mathrm{~m}(325 \mathrm{ft})$

Gross Liftoff Mass:

$907,185 \mathrm{~kg}(2.0 \mathrm{M} \mathrm{lbm})$

25.6 MT (56.5k lbm)

to LEO

(302k lbm)

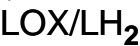

5-Segment

Reusable

(RSRB)

\section{Ares V}

Height: $109.7 \mathrm{~m}$ (360 ft)

Gross Liftoff Mass:

$3,374,910 \mathrm{~kg}(7.4 \mathrm{M} \mathrm{lbm})$

55.9 MT (123k lbm) to Direct TLI -143.4 MT (316k lbm) to LEO
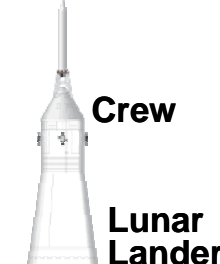

Lander

S-IVB

(1 J-2 engine)

$108,862 \mathrm{~kg}$ (240k lbm) LOX/LH ${ }_{2}$

S-II

(5 J-2 engines)

$453,592 \mathrm{~kg}$

(1M lbm)

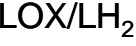

S-IC

(5 F-1)

$1,769,010 \mathrm{~kg}$

(3.9M lbm)

LOX/RP-1

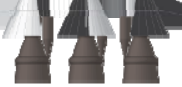

Saturn V

Height: 110.9 m (364 ft)

Gross Liftoff Mass:

2,948,350 kg (6.5M lbm)

$45 \mathrm{MT}(99 \mathrm{k} \mathrm{lbm})$ to TLI 119 MT (262k lbm) to LEO 


\section{Current Ares V 10 meter Shroud}

Shroud Dimensions

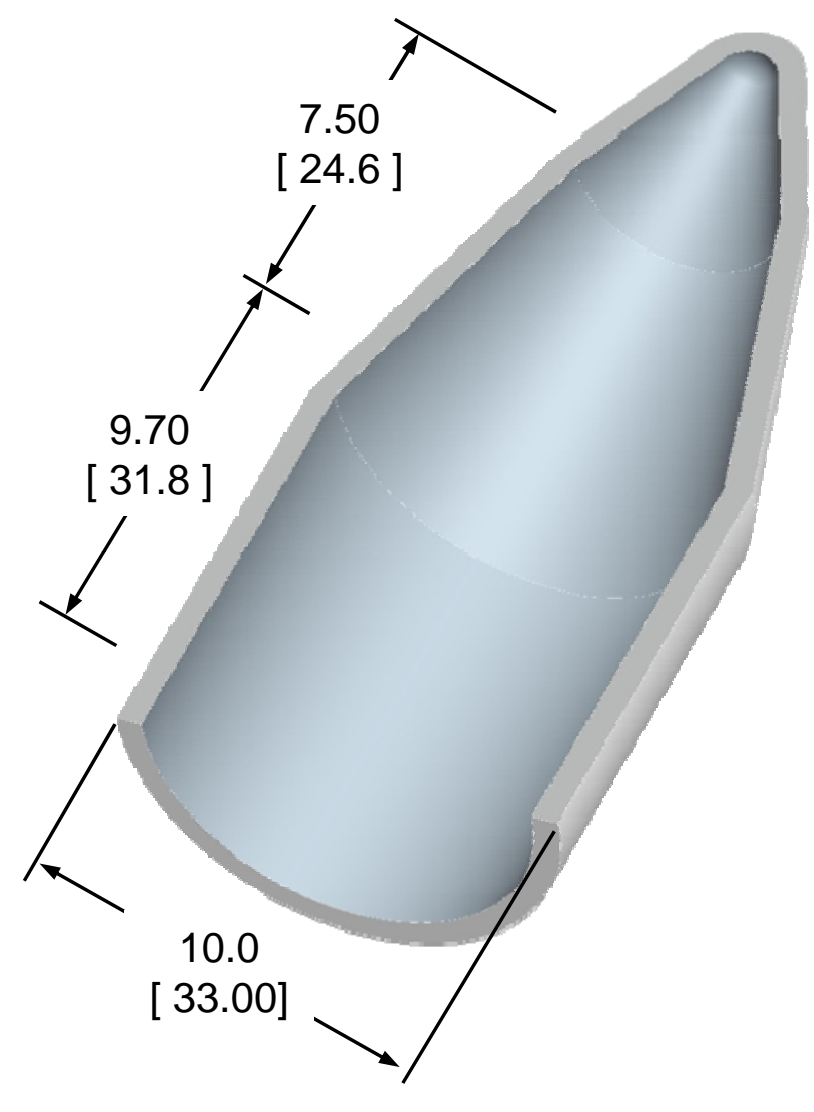

\section{Usable Dynamic Envelope}

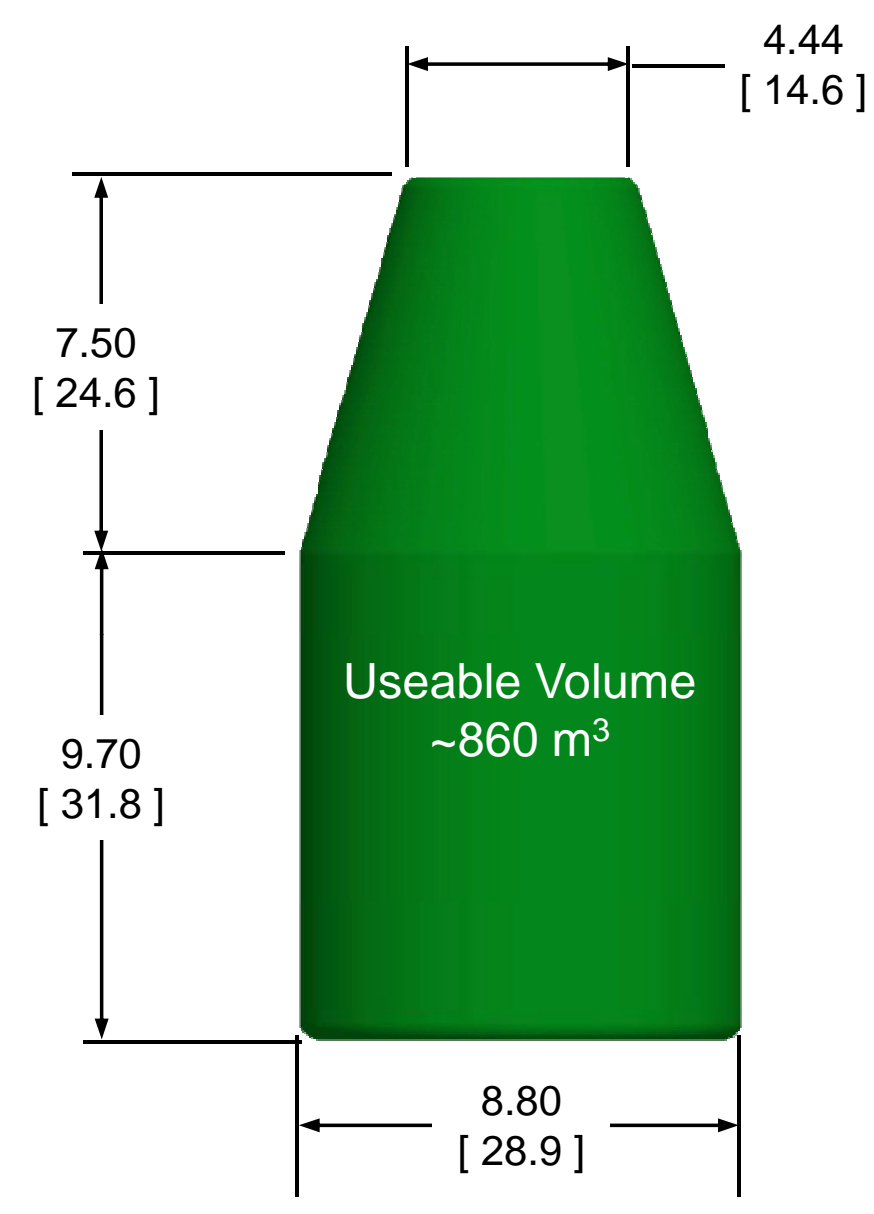




\section{Notional Ares V Shroud for Other Missions}

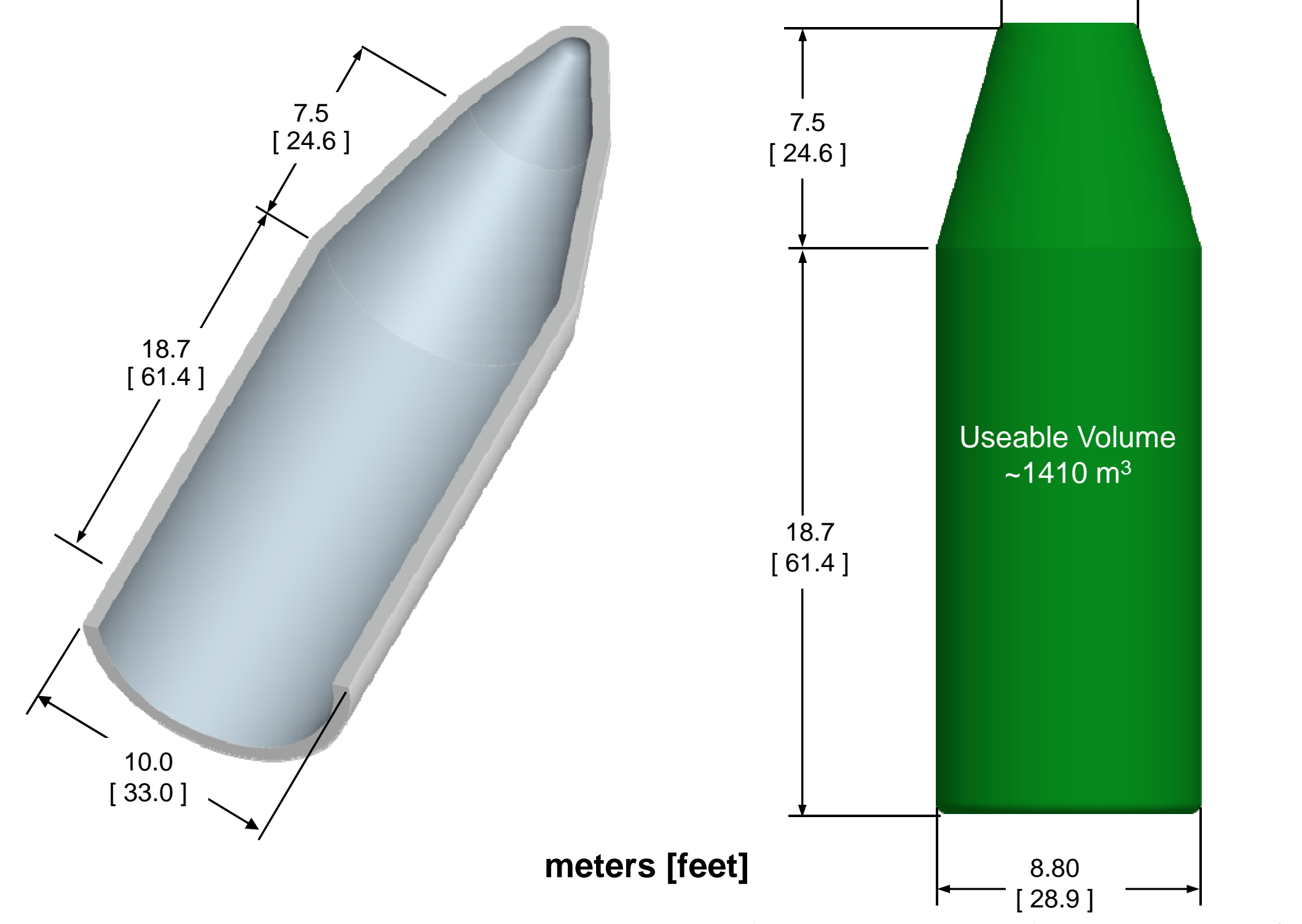

* Note: The height of the shroud is limited by the height of the Vertical Assembly Building (VAB) 


\section{Ares V Performance for Selected Missions}

\begin{tabular}{|c|c|c|}
\hline Mission Profile & Target & $\begin{array}{c}\text { Payload Mass } \\
(\mathrm{kg})\end{array}$ \\
\hline Sun-Earth L2 & $\begin{array}{c}\text { C3 of }-0.7 \mathrm{~km}^{2} / \mathrm{s}^{2} \\
@ 29.0 \mathrm{degs}\end{array}$ & 55,800 \\
\hline GTO Injection & $\begin{array}{c}\text { Transfer DV 8,200 ft/s } \\
\text { Final Orbit: } 185 \text { km X 35,786 km } \\
\text { @ } 27 \mathrm{deg}\end{array}$ & $70,300 *$ \\
\hline GEO & $\begin{array}{c}\text { Transfer DV } 14,100 \mathrm{ft} / \mathrm{s} \\
\text { Final Orbit: } 35,786 \mathrm{~km} \text { Circular } \\
@ 0 \text { degrees }\end{array}$ & 36,200 \\
\hline $\begin{array}{l}\text { Cargo Lunar Outpost (TLI } \\
\text { Direct) }\end{array}$ & $\begin{array}{c}\text { C3 of }-1.8 \mathrm{~km}^{2} / \mathrm{s}^{2} \\
@ 29.0 \mathrm{degs}\end{array}$ & 56,800 \\
\hline
\end{tabular}

* Performance impacts from structural increases due to larger payloads has not been assessed 


\section{Ares V LEO Performance}

Ares V Payload vs. Altitude \& Inclination (LV 51.00.39)

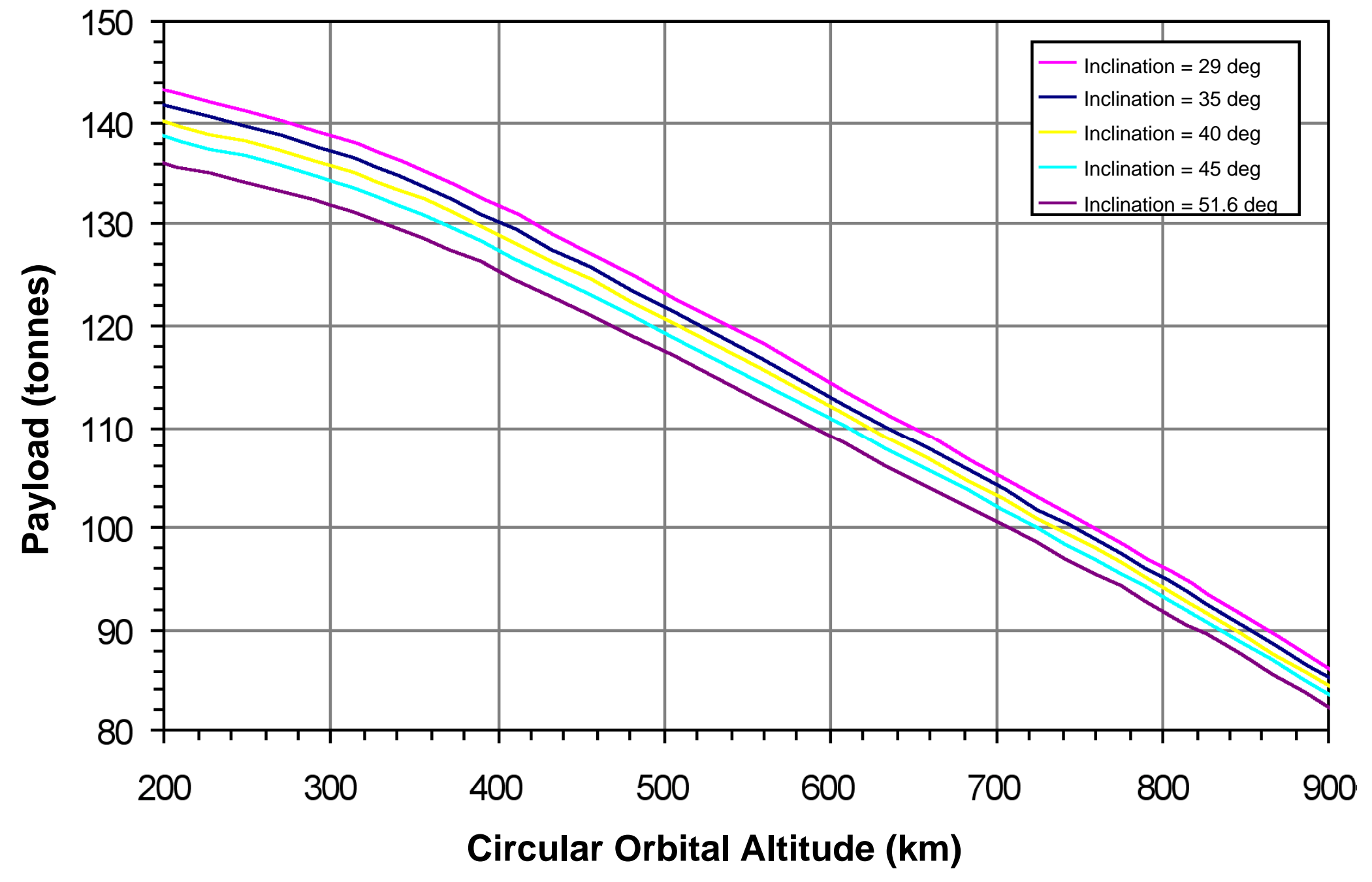




\section{Ares V Escape Performance}

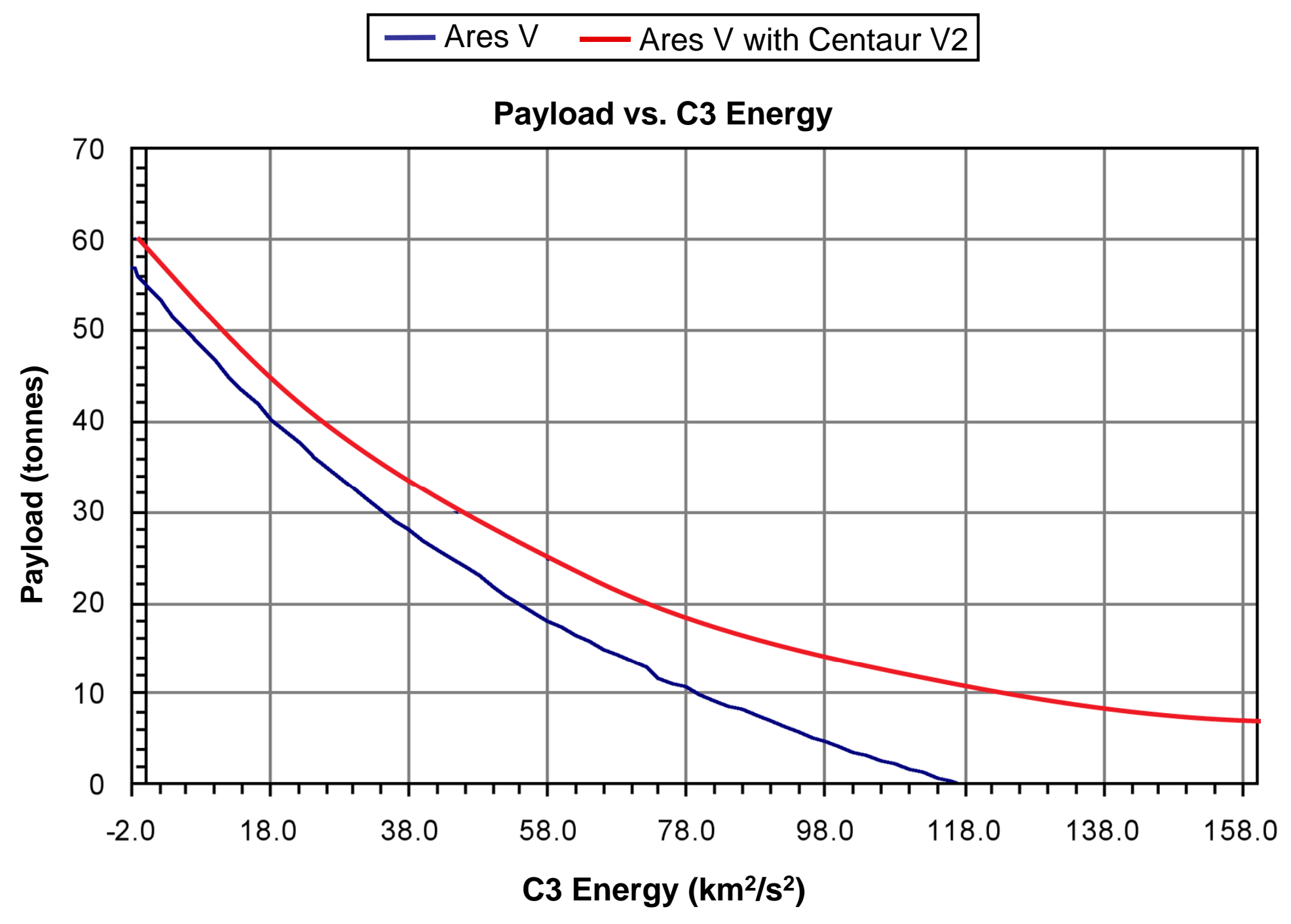




\section{Design study of an 8-meter monolithic mirror UVIoptical space telescope}

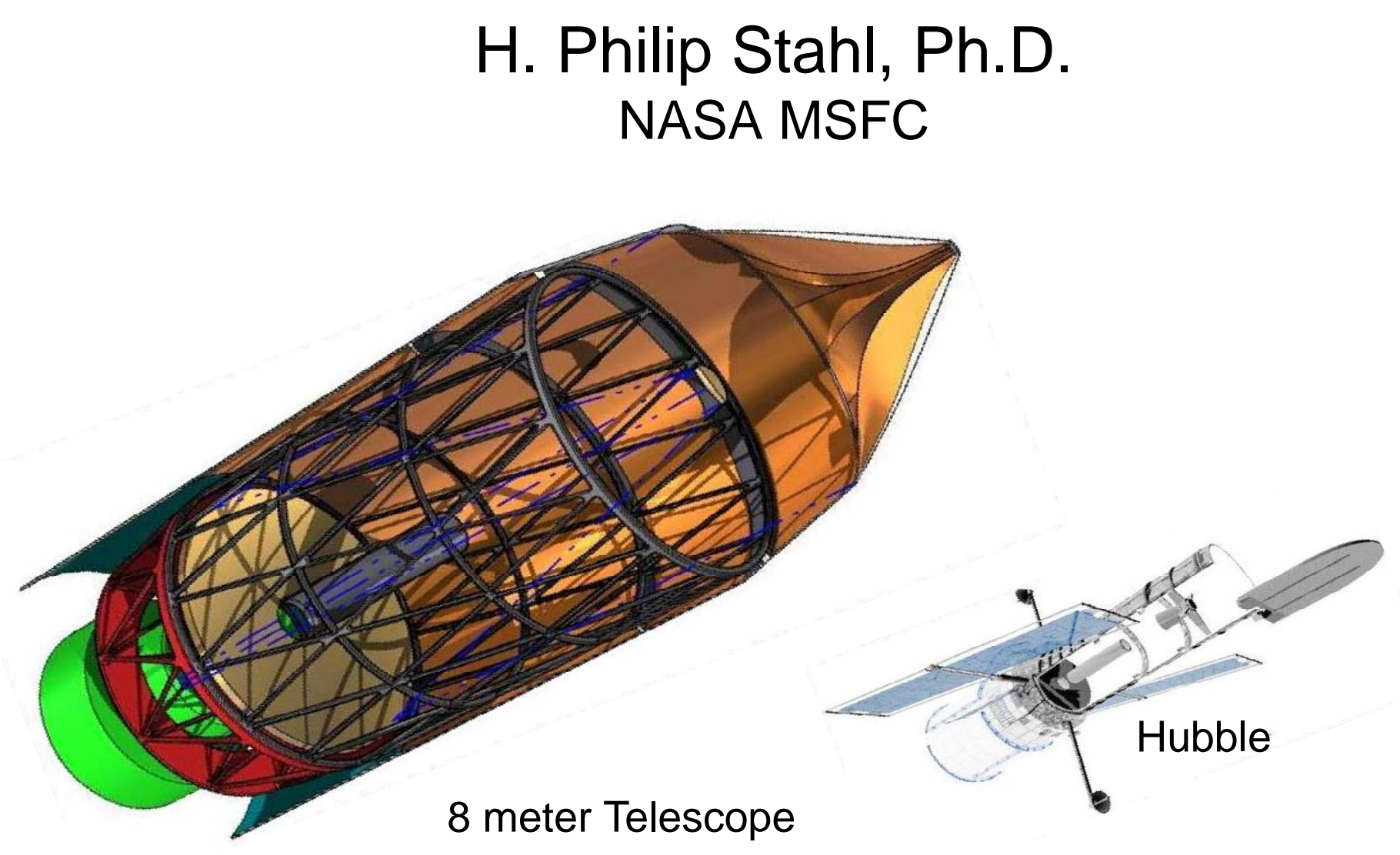




\section{Design Concept}

8 meter Monolithic Telescope \& tube can fit inside Ares $\vee 10$ m envelop.

Minimize Cost (\& Risk) by using existing ground telescope mirror technology - optics \& structure.

8-meter diameter is State of Art

9 existing: VLT, Gemini, Subaru, LBT $23,000 \mathrm{~kg}$ (6 m would be $\sim 13,000 \mathrm{~kg}$ ) $\sim$ \$30M (JWST PM cost $\sim$ \$120M) $7.8 \mathrm{~nm}$ rms surface figure ( TPF spec)

Expect similar savings for structure

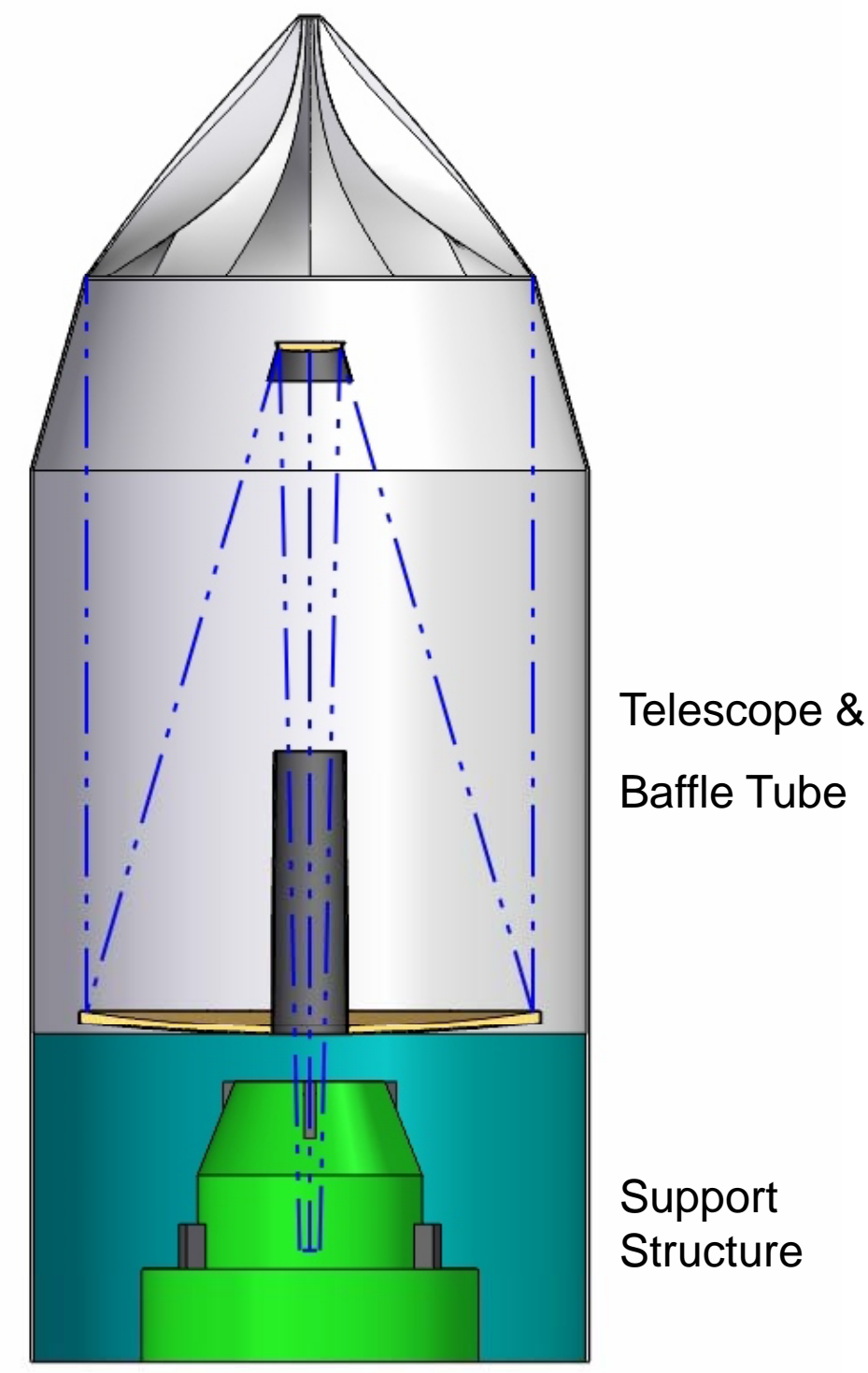




\section{6 meter Optical Design}

Ritchey-Chretién optical configuration

F/15

Diffraction Limited Performance at $<500 \mathrm{~nm}$ Diffraction Limited FOV of 1.22 arc minute (10 arc minute FOV with Corrector Group) Coating: Aluminum with Mg F2 overcoat

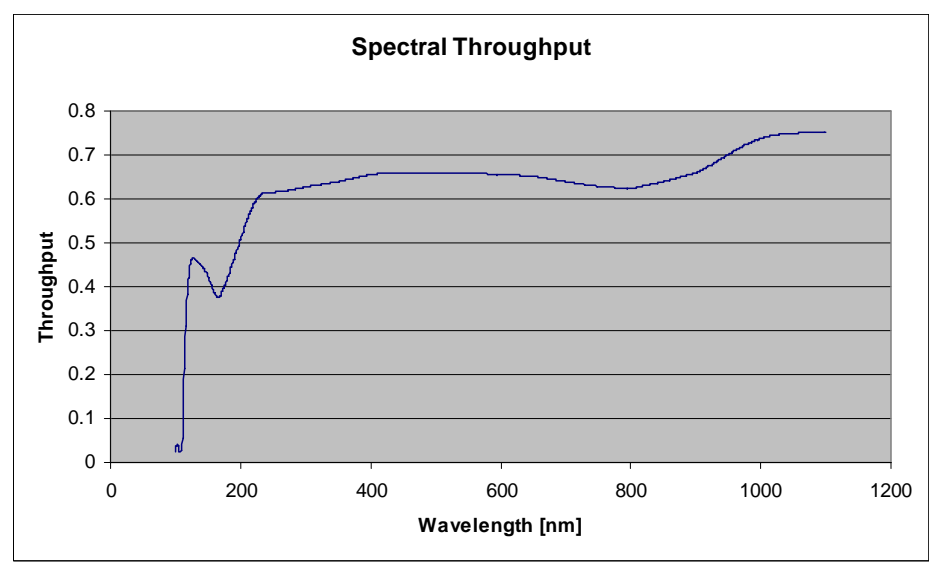

Average transmission $>63 \%$ for wave lengths of 200 to $1,000 \mathrm{~nm}$ Primary to secondary mirror vertex: $9089.5 \mathrm{~mm}$ Primary mirror vertex to focal plane: $3,000 \mathrm{~mm}$

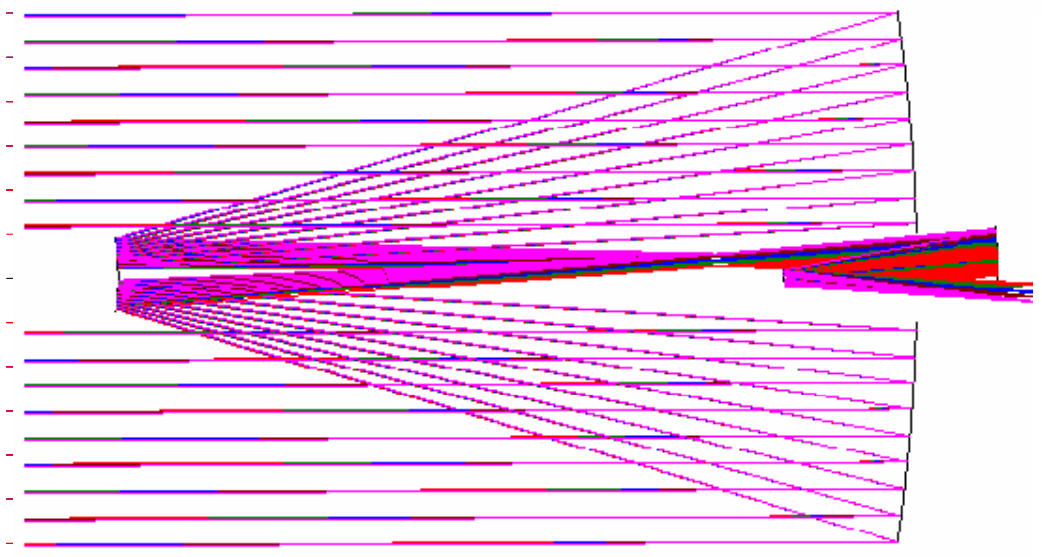

All Reflective Design

Three Mirror Anastigmatic

With Fine Steering Mirror

Multi-Spectral 10 arc min FOV

Reduced Throughput 


\section{Structural Design}

Operational

Launch Configuration

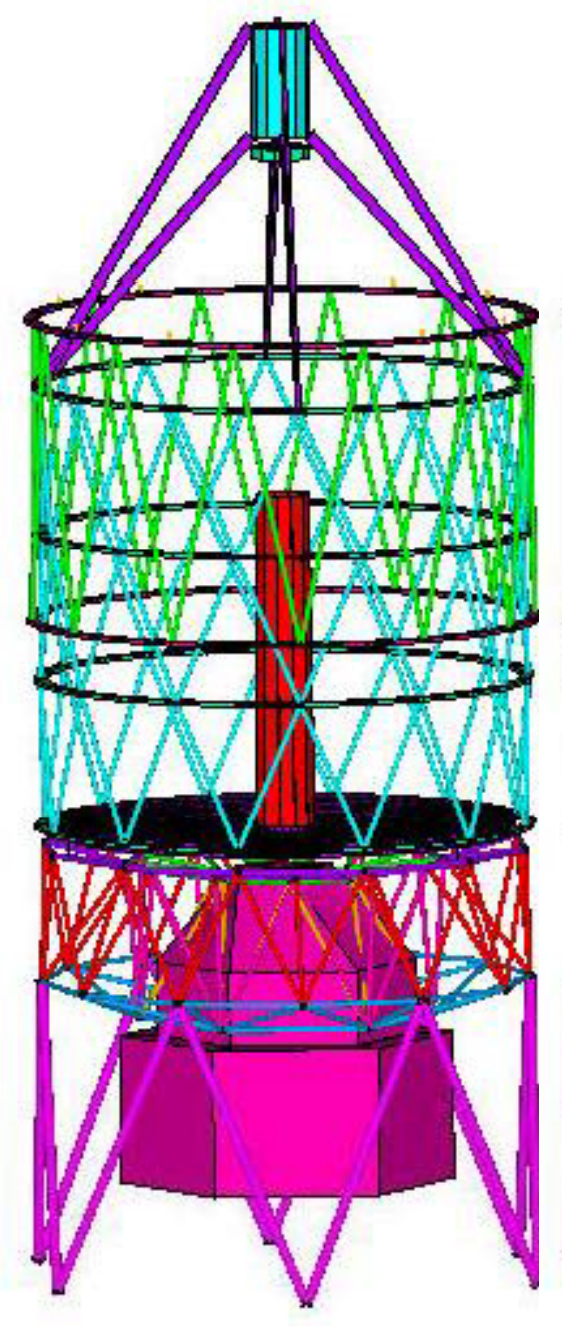

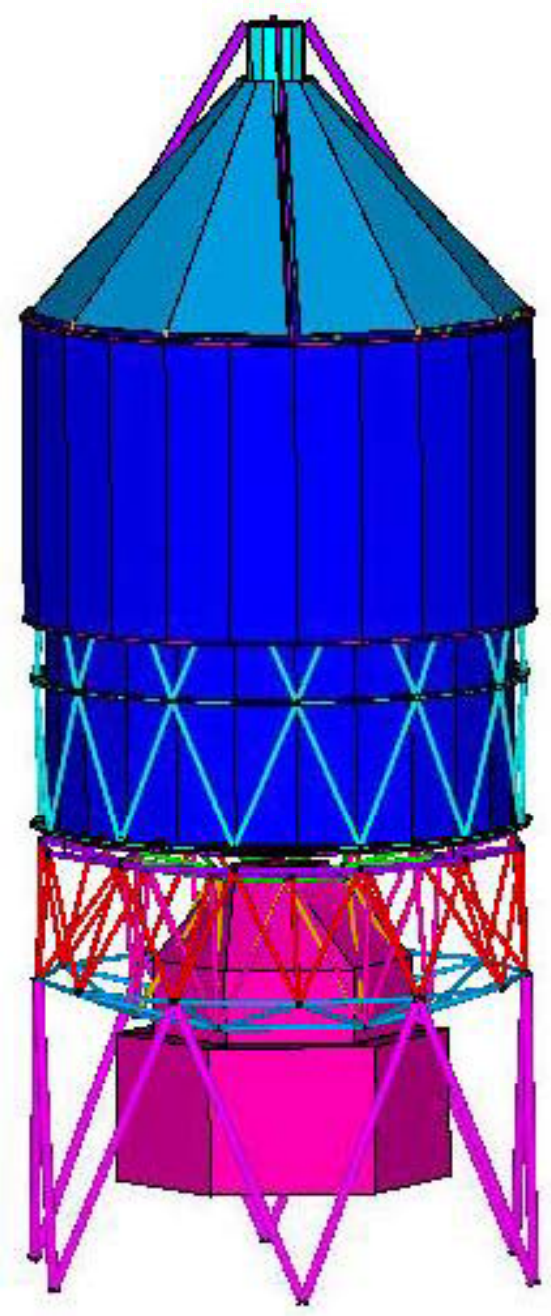

Tube is split and slides forward on-orbit.

Faster PM or taller shroud may allow for one piece tube.

Doors can open/close

Forward Structure is hybrid of Hubble style and four-legged spider

Truss Structure interfaces with 66 mirror support attachment locations

Launch Structure attaches Truss to Ares V
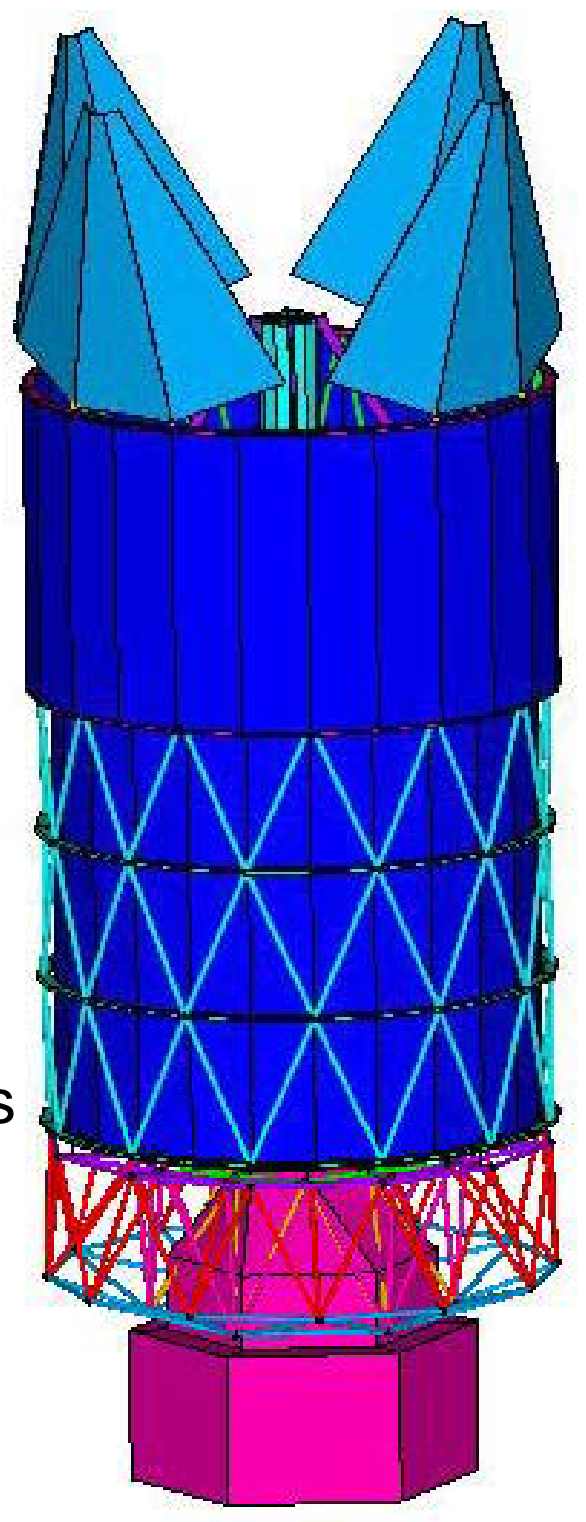


\section{Structural Analysis}

Launch loads: maximum values from POST3D (not concurrent)

$\begin{array}{ll}\text { Axial: } & 4 \mathrm{~g} \text { 's } \\ \text { Lateral-y: } & 7 \times 10^{-6} \mathrm{~g}^{\prime} \mathrm{s} \\ \text { Lateral-z: } & 6 \times 10^{-4} \mathrm{~g}^{\prime} \mathrm{s}\end{array}$

8.2 meter $175 \mathrm{~mm}$ thick meniscus primary mirror can survive launch. 66 axial supports keep stress levels below 1000 psi
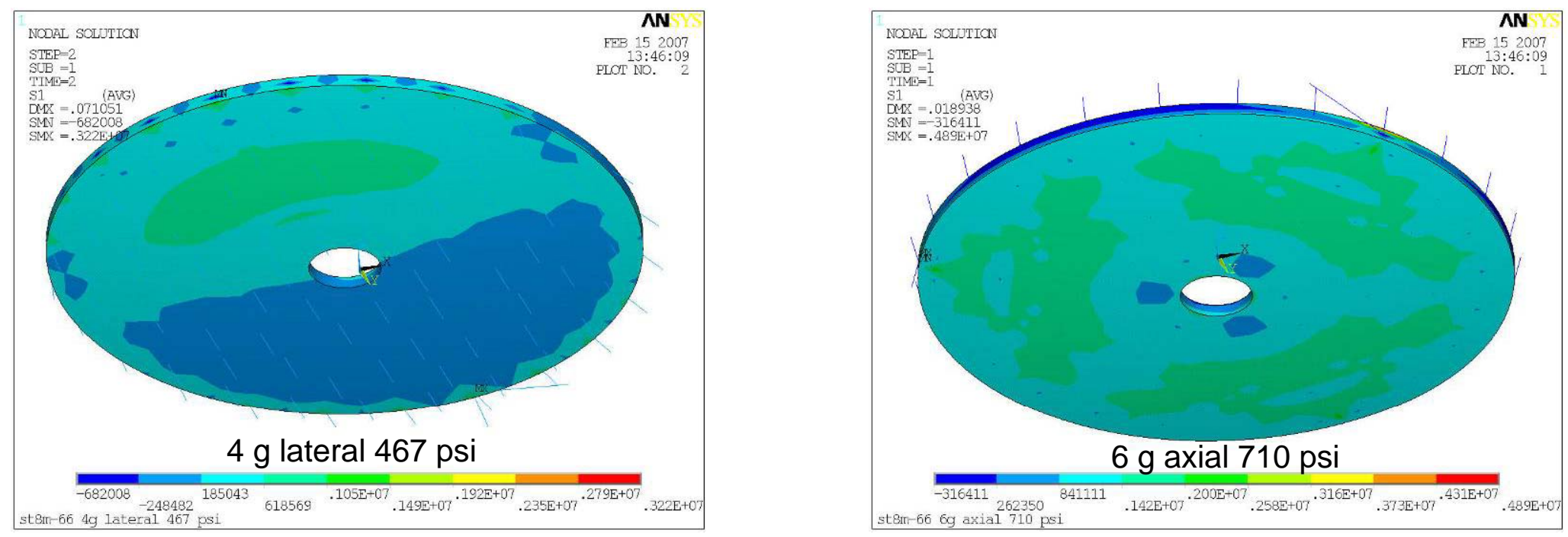


\section{Spacecraft Structural Modeling}

Instrument Frame \& Outer Skin Not Shown

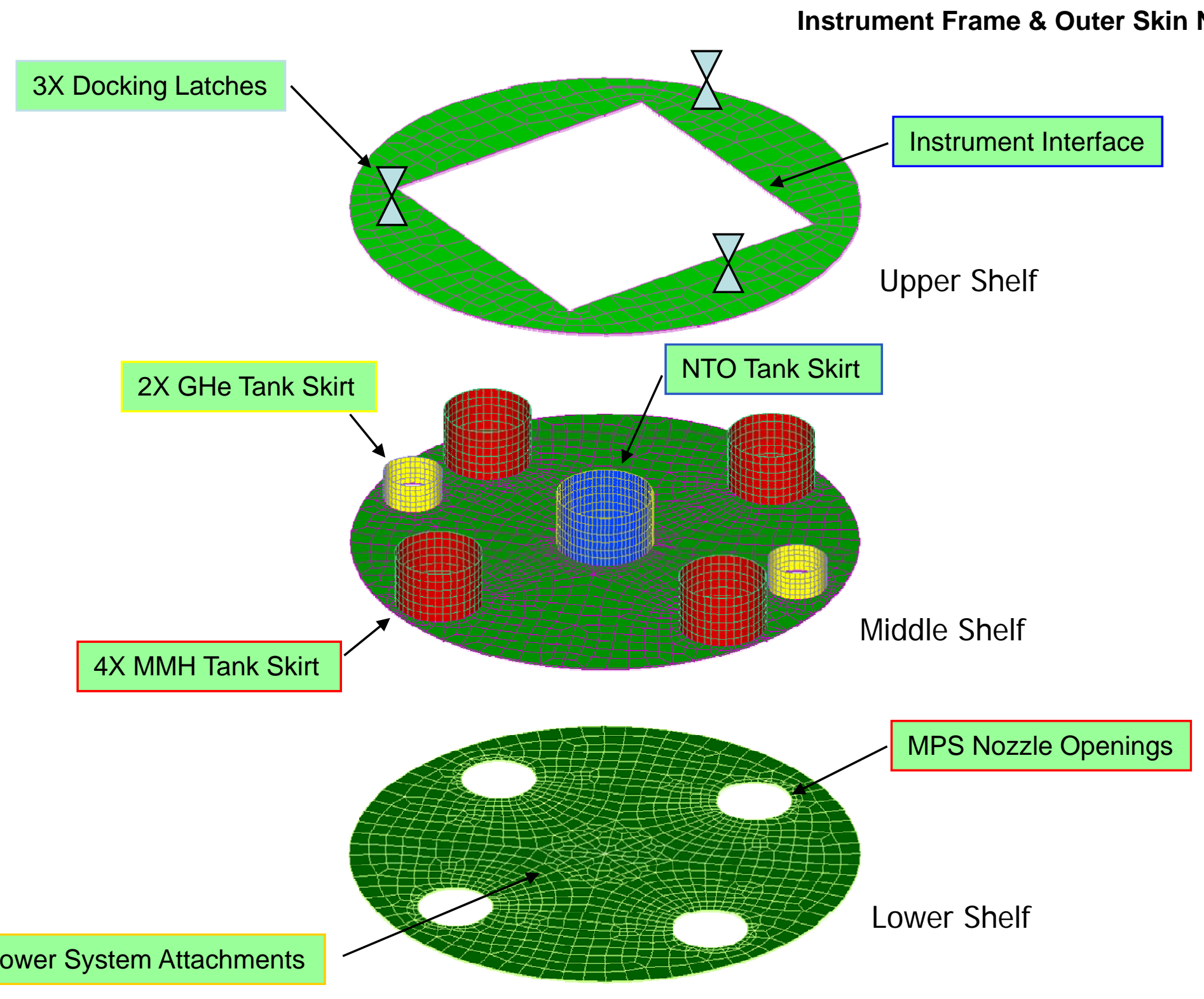




\section{Spacecraft Structural Analysis Assumptions}

Launch Load Case: 4.0g Axial + 2.0g Lateral

Materials: Metallic Structure Only

AA 2219 for plate elements

AL 7075 for Beam Elements

Factors of Safety: (per NASA-STD-5001)

Yield Factor of Safety: 1.1

Ultimate Factor of Safety: 1.4

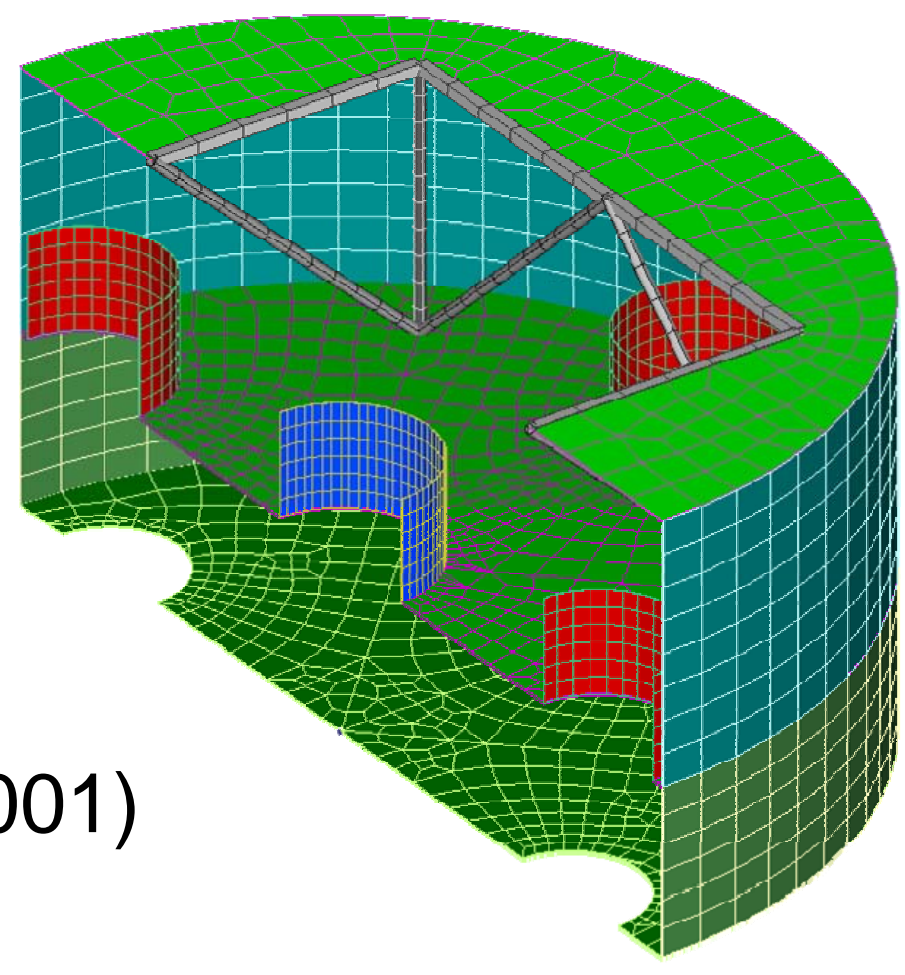

Cross-Sectional View of Spacecraft 


\section{Structural Model Results}

\section{Upper Shelf:}

Shelf: Isogrid Panel 0.090" (minimum pocket thickness)

Middle Shelf:

Shelf: Isogrid Panel 0.060" (minimum pocket thickness) MMH Skirts: 0.064" thk NTO Skirt: 0.088" thk GHe Skirt: 0.040" thk

Lower Shelf:

Shelf: Isogrid Panel 0.060"

(minimum (pocket thickness)

Instrument Support Frame:

Upper Support: "T" Beam, 0.095" thk

Uprights: 2" diameter, 0.030" thk

Angled Supports: 1.75" diameter, 0.030" thk

\section{Outer Skin:}

Upper Outer Skin: 0.26" thk Lower Outer Skin: 0.21" thk

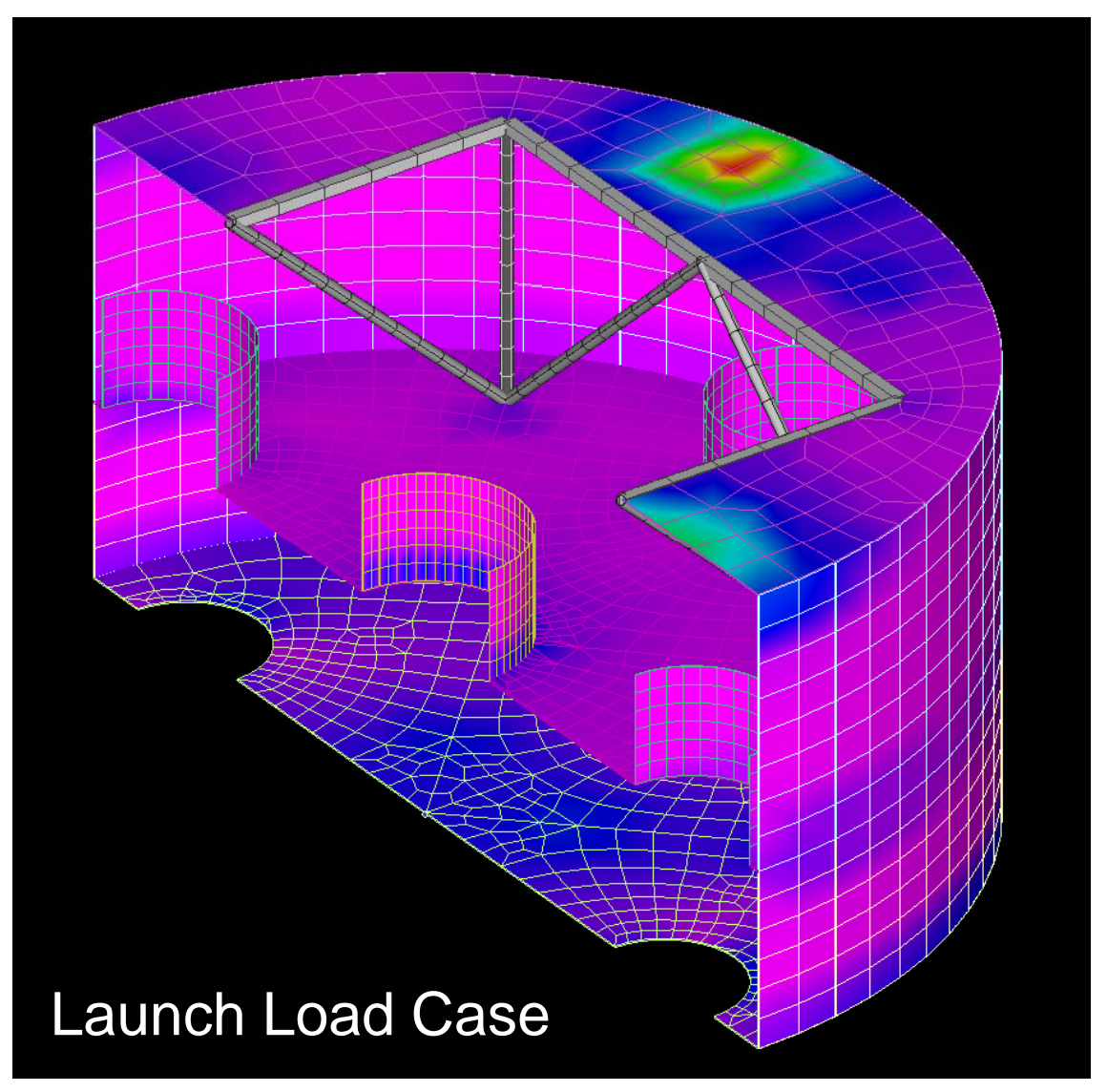




\section{Spacecraft Design Detail \& Shroud Integration}

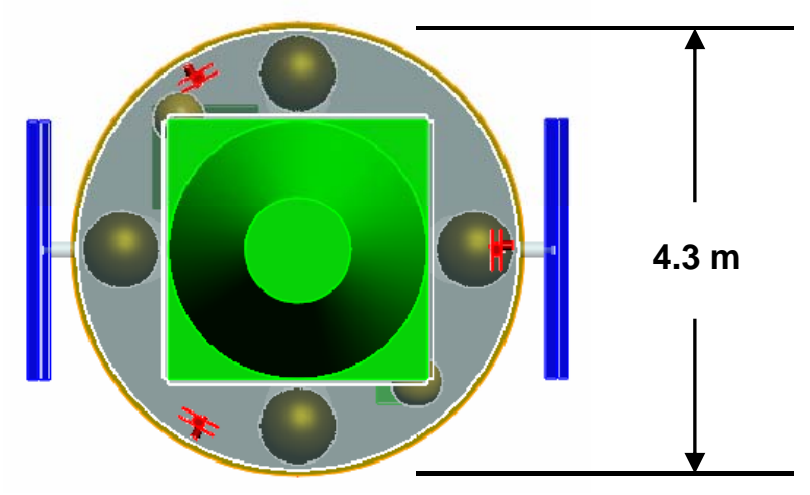

Top View

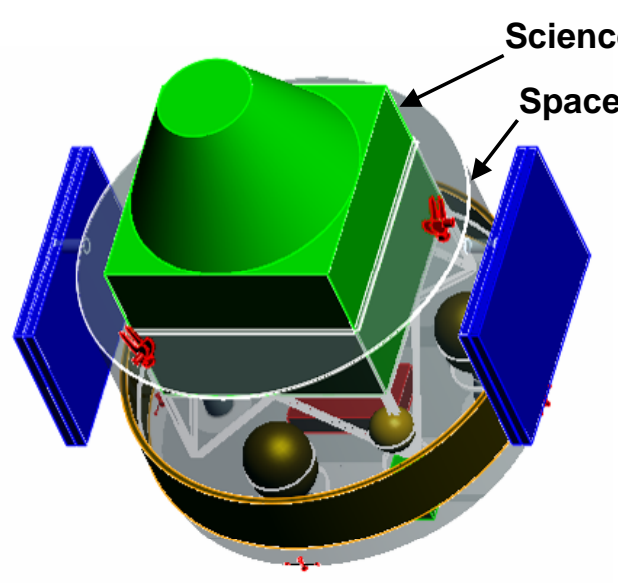

Iso View

Science Envelope $=2.5 \mathrm{~m} \times 2.5 \mathrm{~m} \times 2.0 \mathrm{~m}$

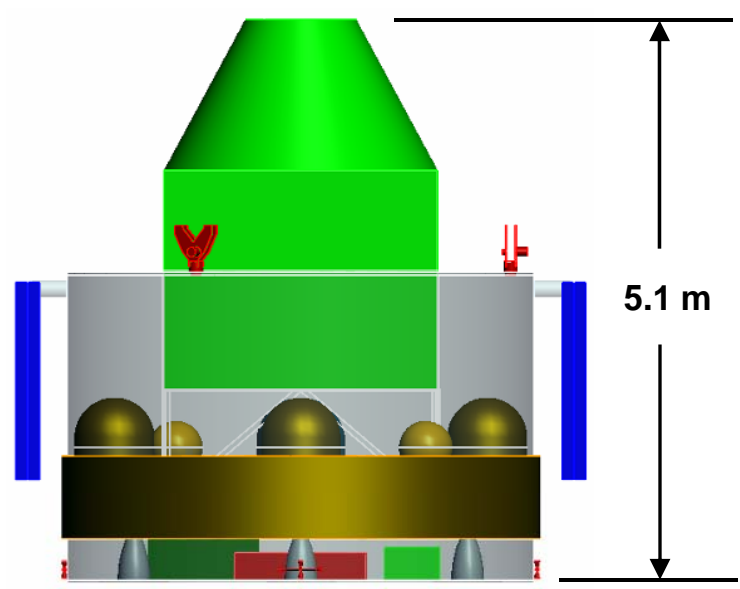

Front View

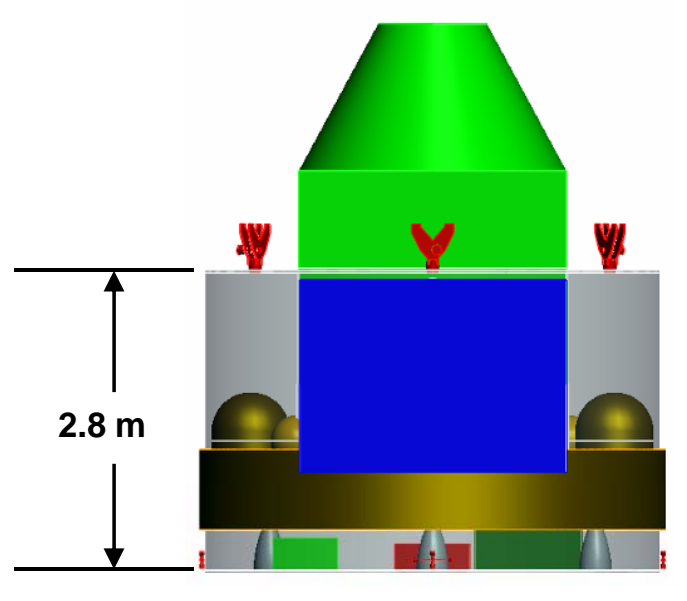

Side View

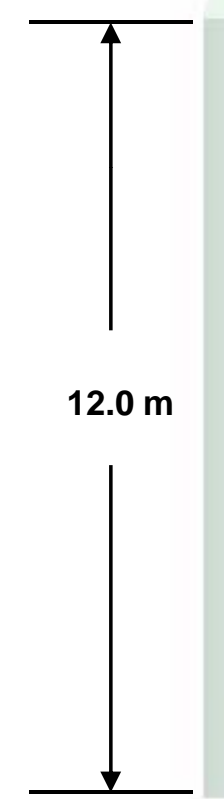

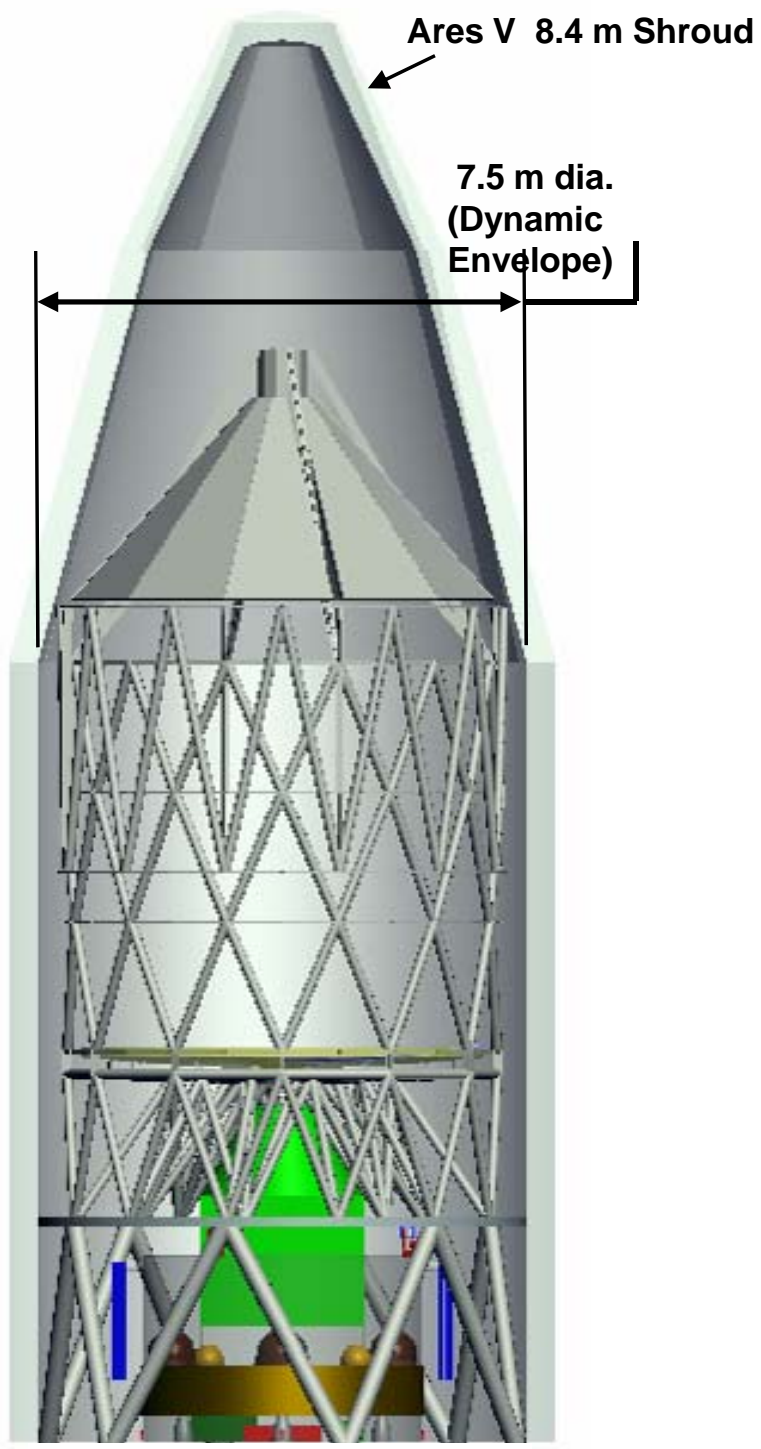

Front View

NOTE: All dimensions are in meters. 


\section{6 meter Preliminary Mass Budget}

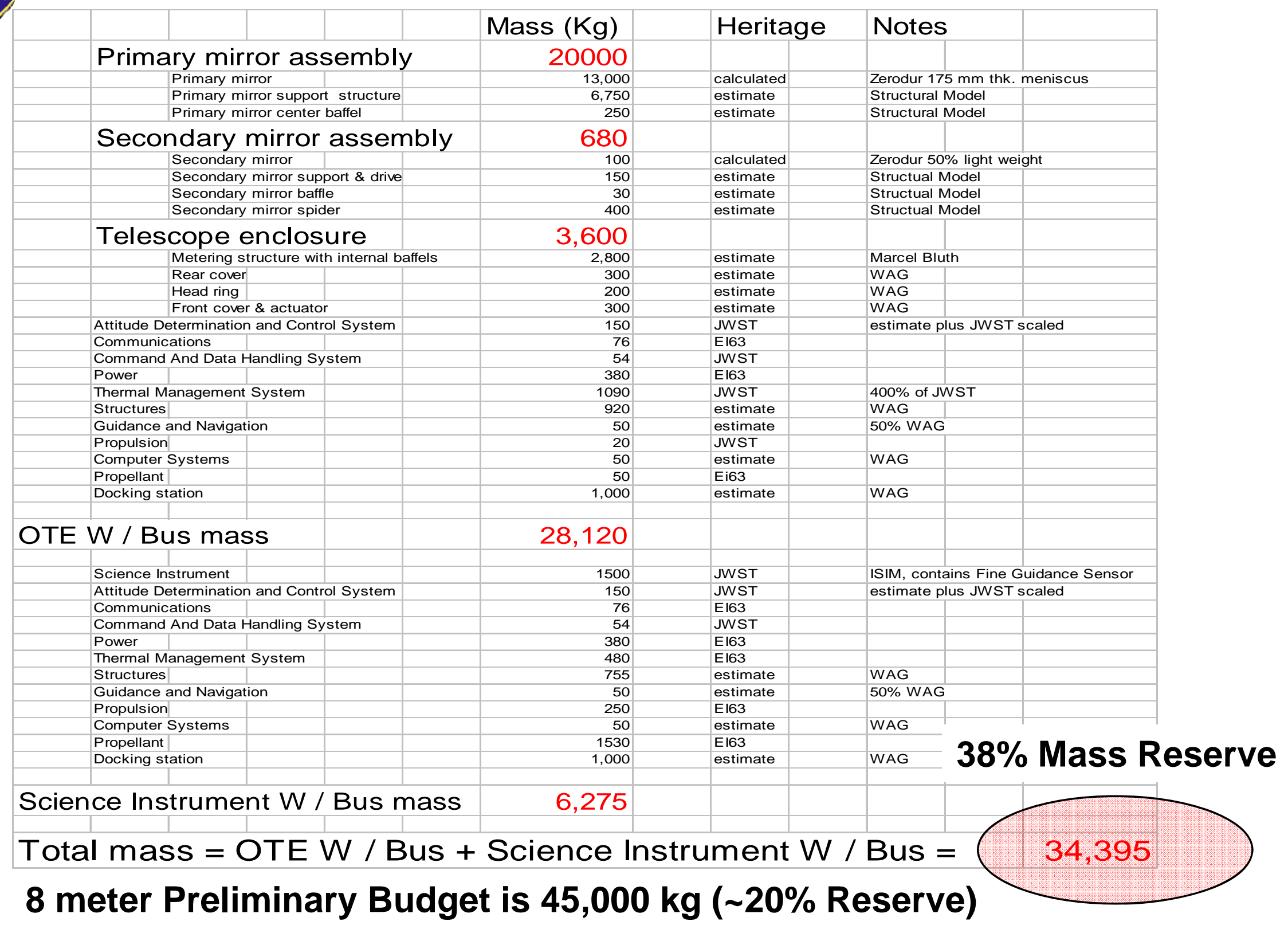




\section{Thermal Analysis}

Spacecraft wrapped with 10 layer MLI blankets

$16.0 \mathrm{~m}^{2}$ thermal radiators

Load Cases
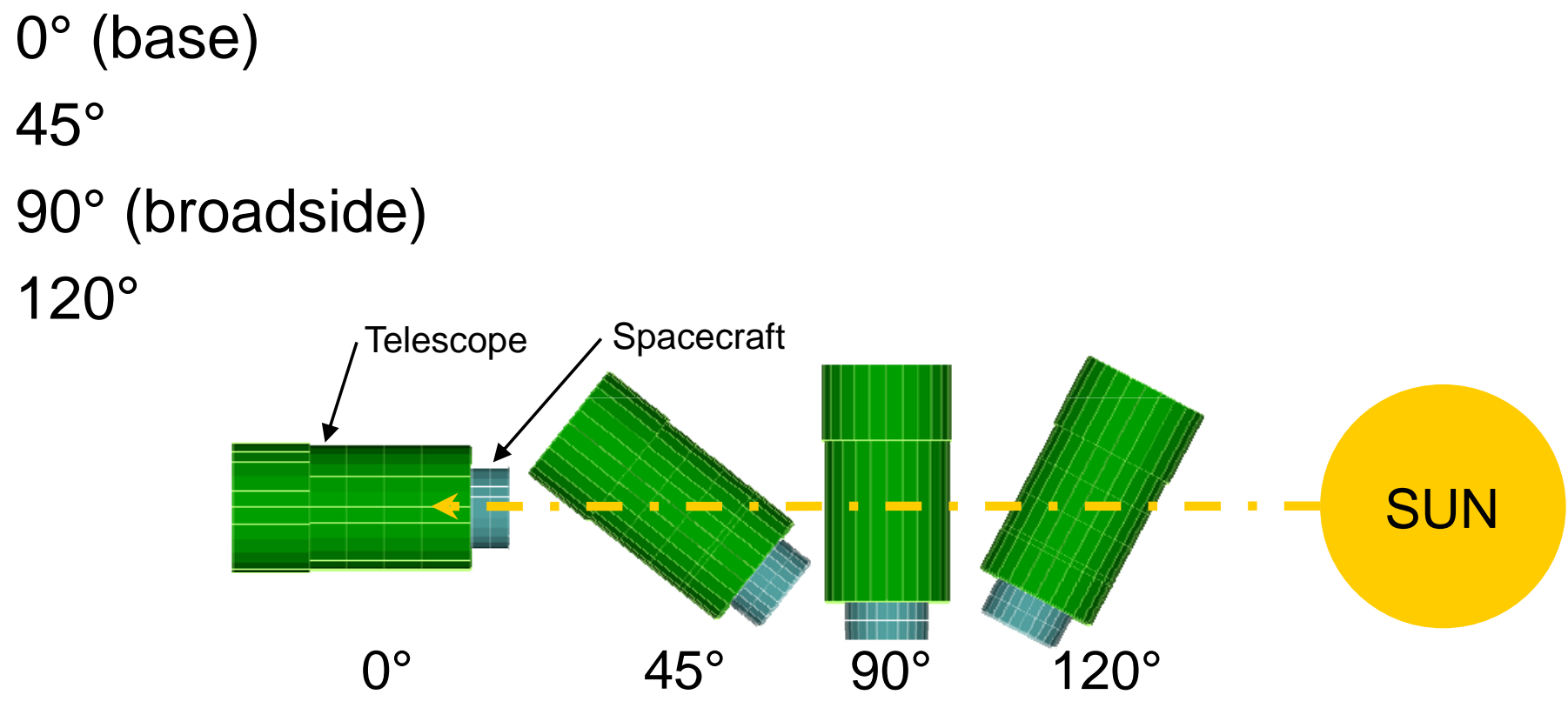


\section{Spacecraft Thermal Analysis}

Solar Flux at L2 $=1296 \mathrm{~W} / \mathrm{m}^{2}$ applied to base Instrument Heat Output $=750 \mathrm{~W}$

Avionics Heat Output $=850 \mathrm{~W}$

Propellant tanks modeled as

single nodes with heat leaks

from the spacecraft walls

Steady-state operational

temperatures determined

Spacecraft wrapped with

50 layer MLI blankets

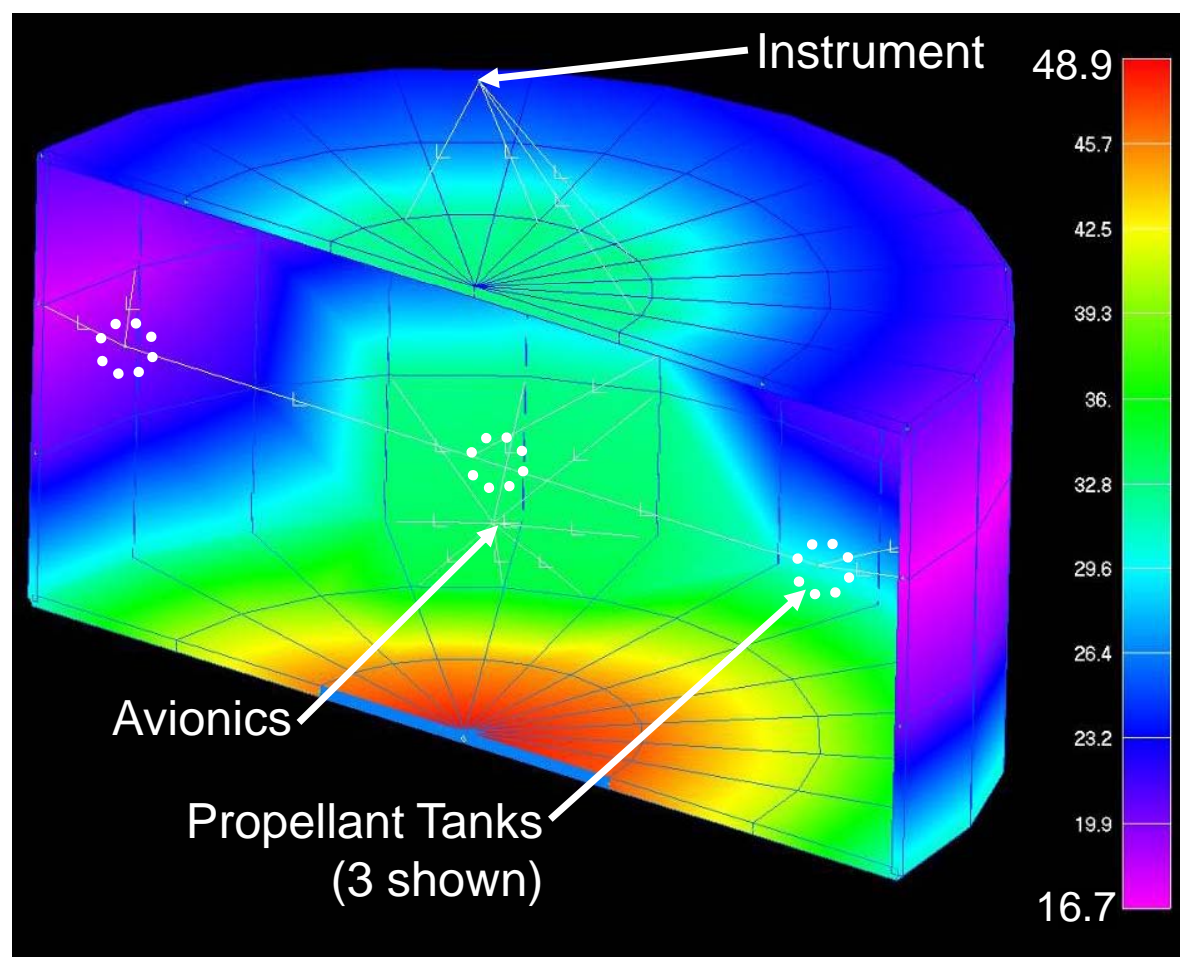

$16.0 \mathrm{~m}^{2}$ thermal radiators

Propellant tanks maintained with $\mathrm{MLI}$ and heaters

Heaters required to keep propellant from freezing 


\section{Primary Mirror Thermal Analysis Results}
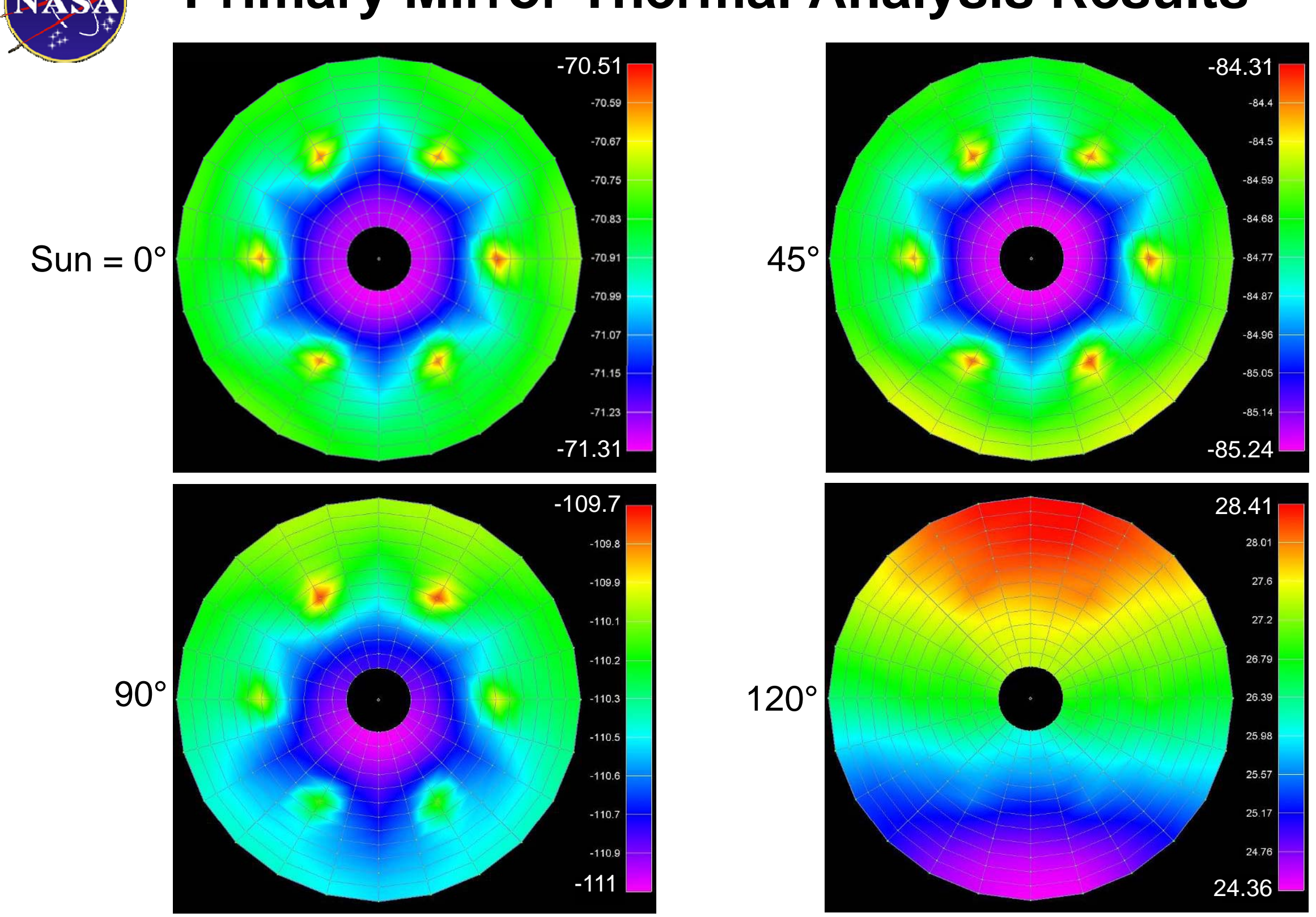

* Temperatures are in ${ }^{\circ} \mathrm{C}$. Note varied temperature scale for each load case. 


\section{Primary Mirror Thermal Analysis}

Active Thermal Management via 14 Heat Pipes yields a Primary Mirror with less than $1 \mathrm{~K}$ Thermal Variation.

No Thermal Management yields a Cold PM

Sun Angle

0 deg

$90 \mathrm{deg}$

$120 \mathrm{deg}$

with $1 \mathrm{~K}$ Thermal Variation

Thus, possible End of Life use as a

NIR/Mid-IR Observatory.

Figure Change will be driven by CTE Change from $300 \mathrm{~K}$ to $150 \mathrm{~K}$

Zerodur CTE is approximately $0.2 \mathrm{ppm}$. SiO2 CTE is approx $0.6 \mathrm{ppm}$.
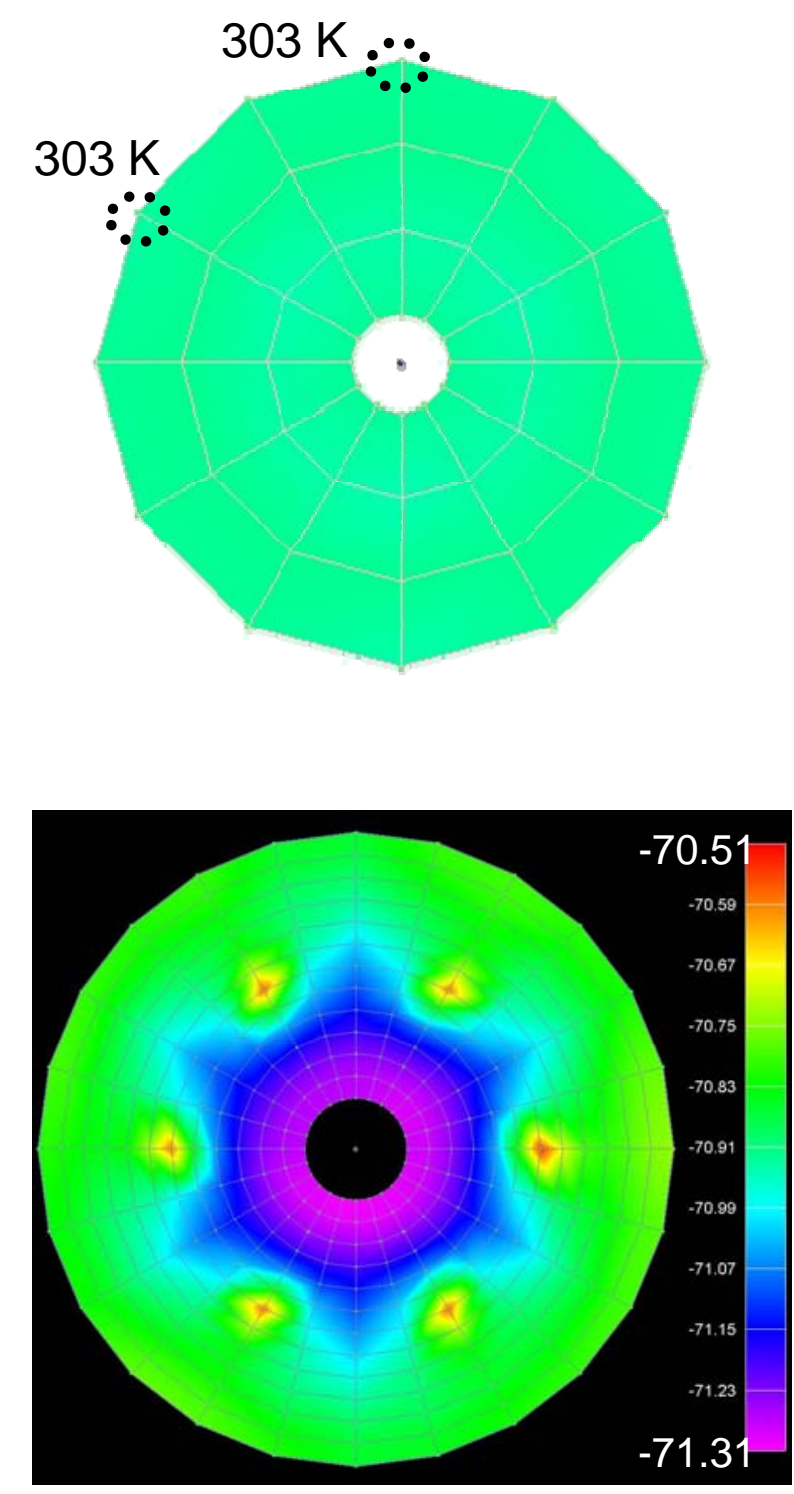


\section{Notional Spacecraft Propulsion System}

Dual Mode: Hydrazine-NTP Bi-Prop / Hydrazine Mono-Prop

Propellant for $5 \mathrm{yr}$ mission with redundant Thrusters

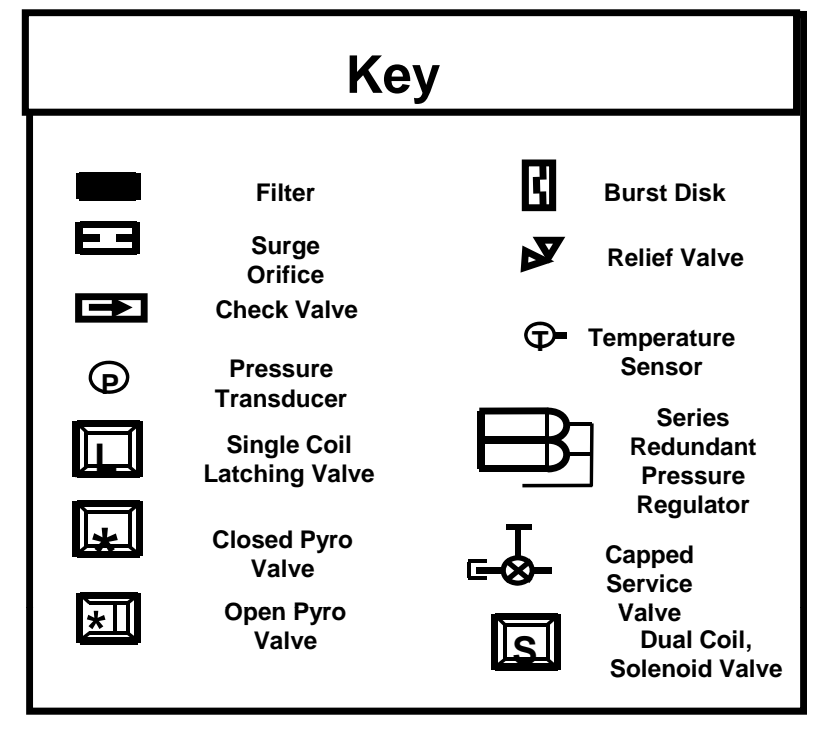

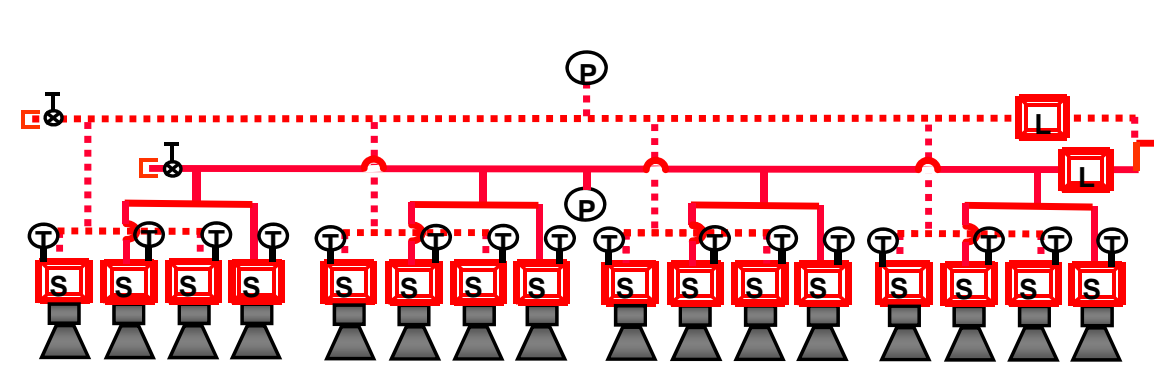

Hydrazine Mono-Prop with RCS 20/5 lbf Thrusters (Aerojet) for Station Keeping

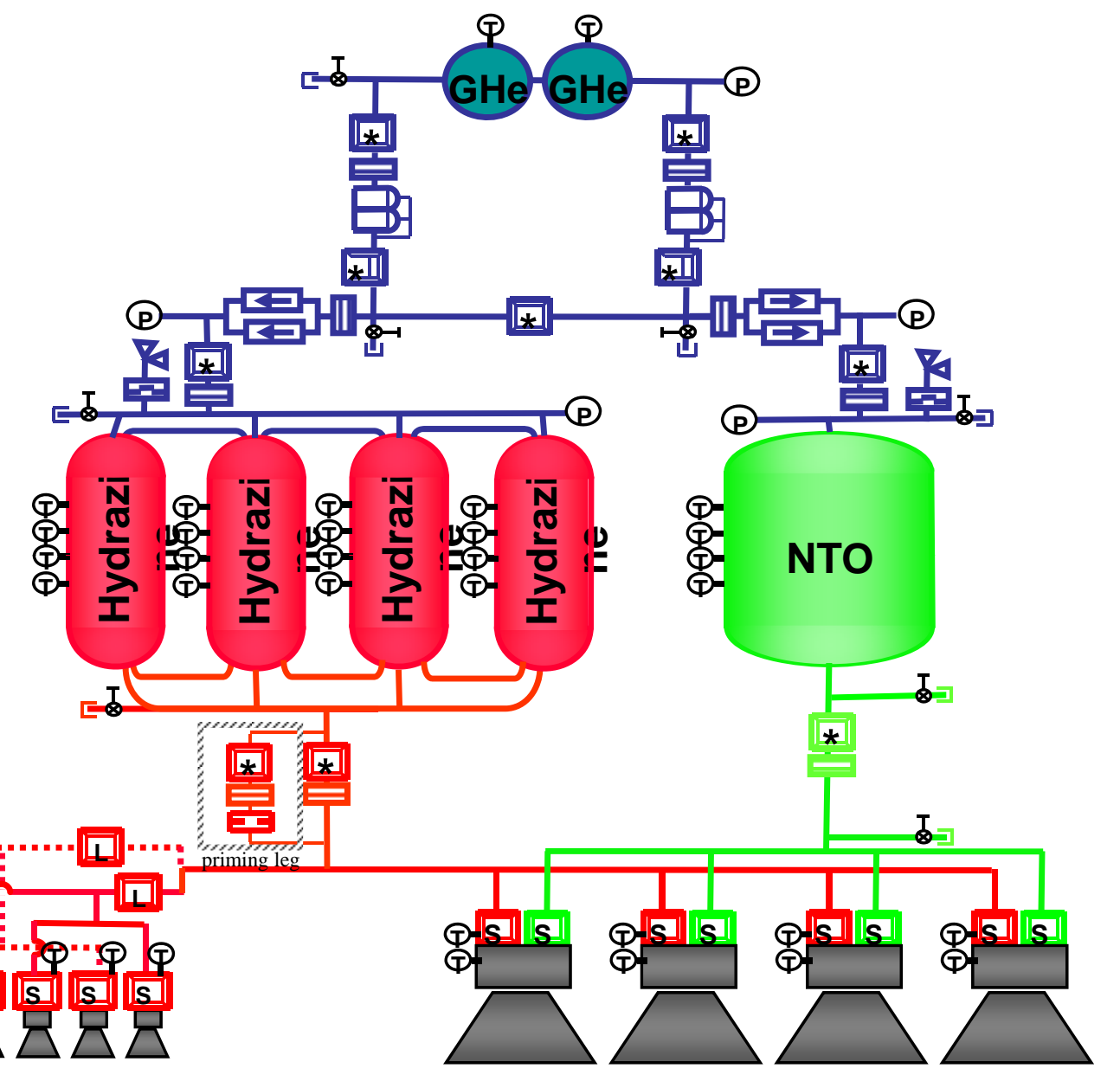

Hydrazine-NTP Bi-Prop with four $125 \mathrm{lbf}$ Thrusters (Northrop) for trip to L2 


\section{Notional Telescope Propulsion System}

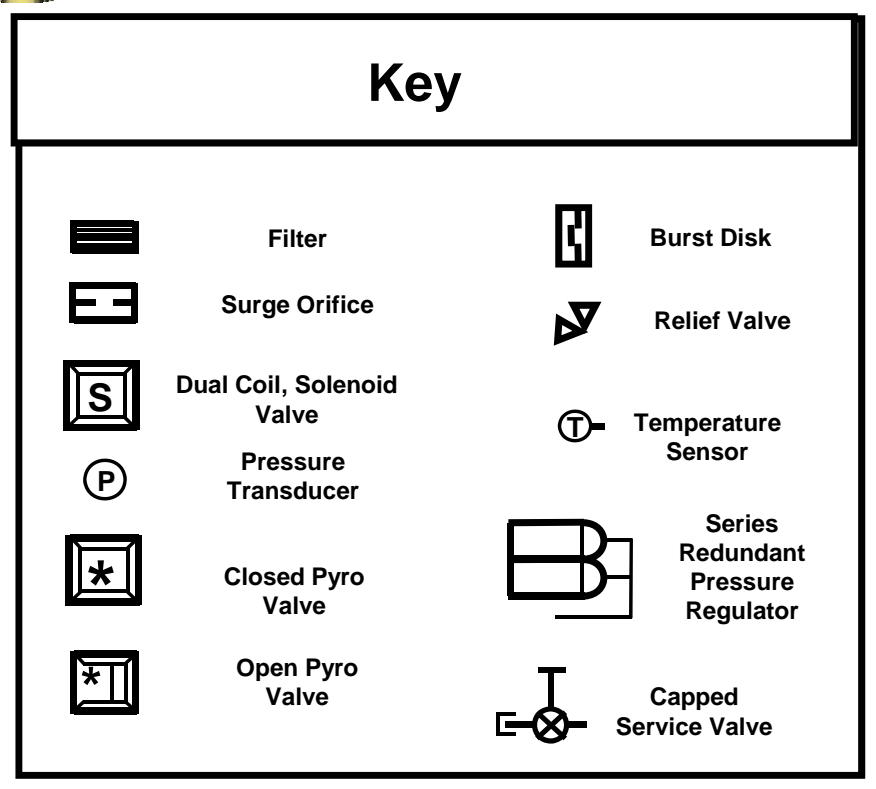

Telescope has Independent Control System

Mono-Propellant Hydrazine

Trade Analysis:

Refueling (Orbital Express) $=40 \mathrm{~kg}$

30 Year Propellant Supply $=30 \mathrm{~kg}$

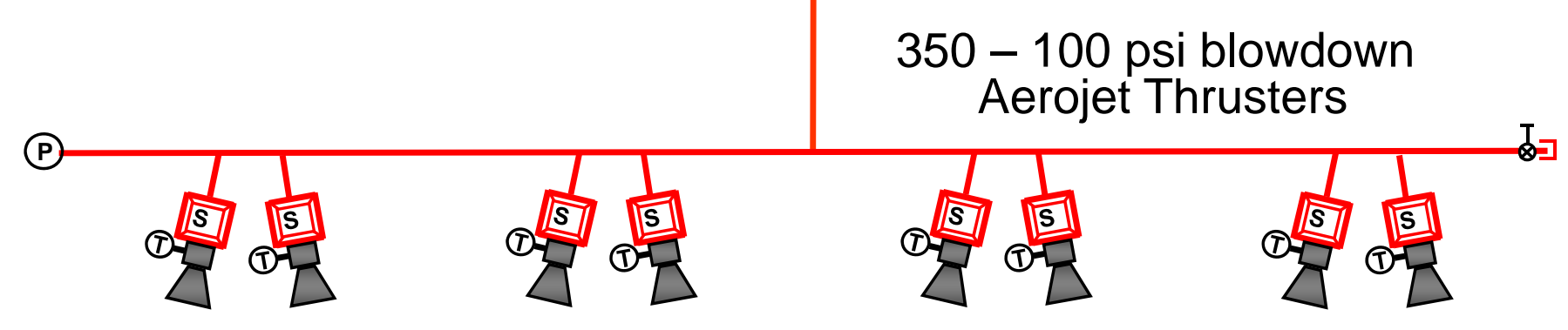




\section{Guidance Navigation and Pointing Control}

Spacecraft Reaction Wheels provide all GNC

Worst condition for solar radiation pressure torque is at sun angle $=90$.

Momentum buildup occurs in one axis (y-axis)

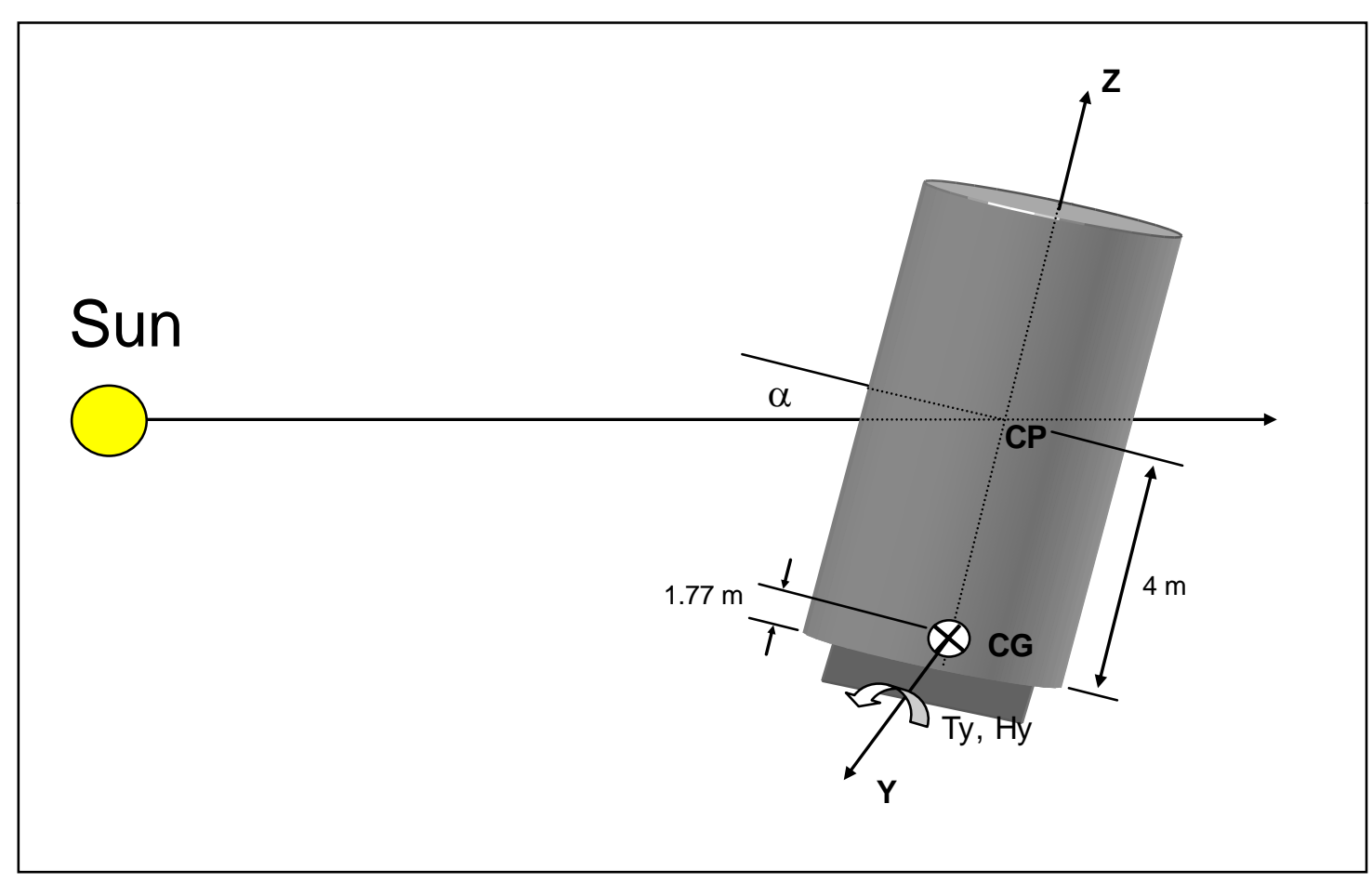




\section{GN\&C Analysis}

Two performance Parameters were analyzed and plotted against each other:

- Hours that Telescope can stare at a fixed point (remain at an inertial hold) before needing to perform a momentum dump due to solar radiation pressure torque

- How fast in minutes the Telescope can perform a 60 degree slew

6 wheel and 4 wheel configurations were analyzed along with the worst case single wheel failure for each configuration.

Each configuration was analysis for three different TELDIX reaction wheel versions with different (Torque : Momentum Storage)

Analysis

is only for the worst case sun angle $=0$

As the sun angle increases so does the available science time. did not account for any solar panel contribution to solar pressure cp location.

This is worst case since accounting for the solar panels would move the cp location closer to the cg. Also, Telescope geometry is preliminary and may change due refinement in design 


\section{GNC: Reaction Wheels}

Science Time vs Slew Time

6 and 4 Reaction Wheel Configurations

(Single Axis Solar Pressure Disturbance Torque and Single Axis Slew)

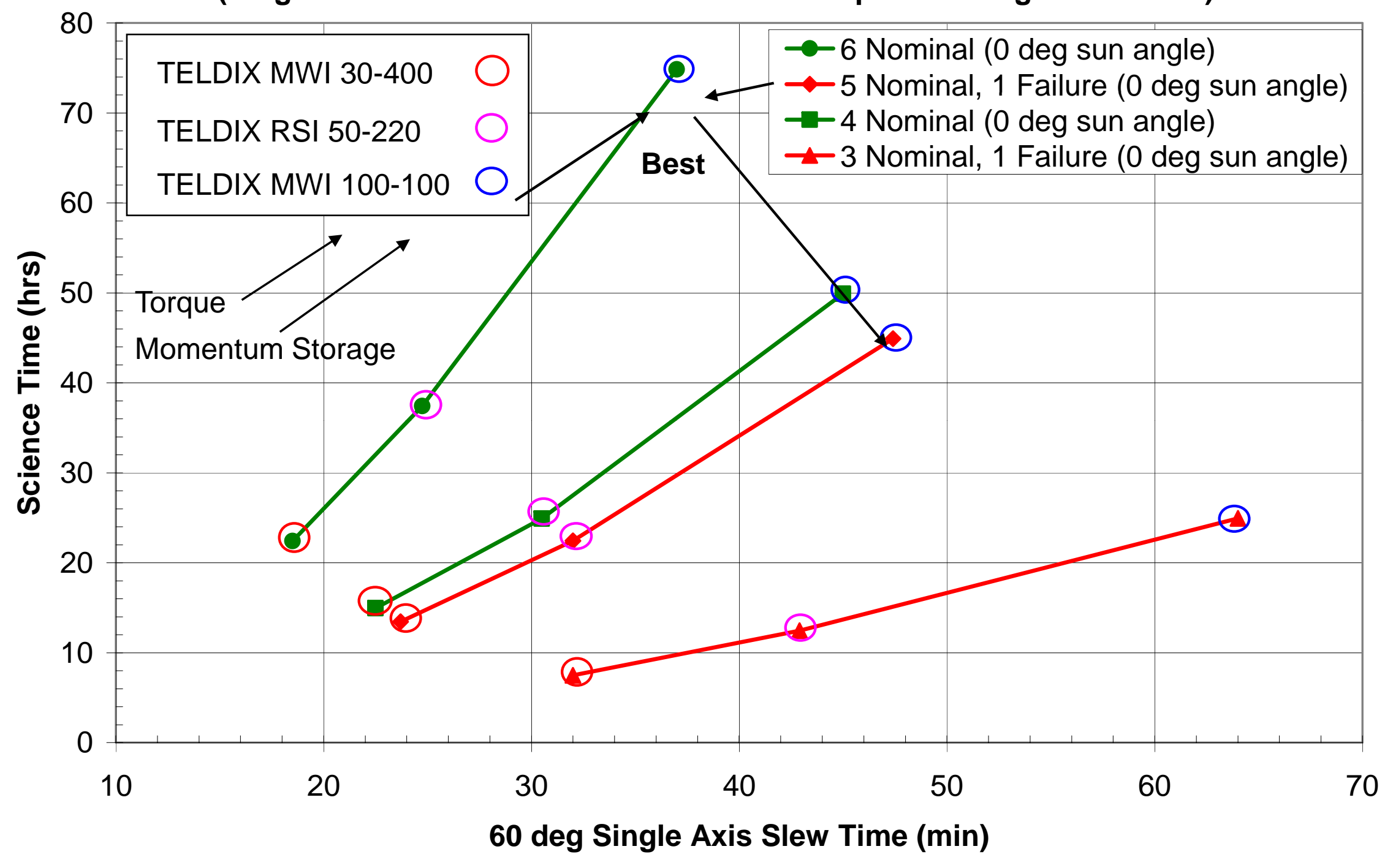




\section{Avionics and Power Systems Assumptions}

\section{Spacecraft}

\section{Avionics}

-Spacecraft avionics systems are 1-fault tolerant for 5 year life

- Guidance and navigation system includes star trackers, sun sensors, and IMUs

-AR\&D consists of a LIDAR long range system, and an optical short range system

-Computers handle all normal station keeping, maneuvers, data management, and ground communications

-Communication systems consist of Ka-band HGA for ground, and s-band for local comm and backup capability

\section{Power}

-Spacecraft power systems are 1-fault tolerant for 5 year life

- Power generation from two $9 \mathrm{~m}^{\wedge} 2$ deployable solar array wings with pointing ability -Batteries are sized for 2 hours of power for midcourse and rendezvous operations (with arrays retracted)

-Spacecraft power system includes $800 \mathrm{w}$ for mirror thermal control, and $750 \mathrm{w}$ for telescope instrument package 


\section{Avionics and Power Systems Assumptions}

\section{Telescope}

\section{Avionics}

-Telescope avionics systems are 3-fault tolerant for 30 year life

-Minimal guidance and navigation system, used only for station keeping during spacecraft exchange

-Minimal computer capability, used mainly for station keeping during spacecraft

exchange

-All health and status data sent directly to spacecraft avionics system

-Low gain communications capability with the servicing spacecraft only

\section{Power}

-Telescope power systems are 3-fault tolerant for 30 year life

- $18 \mathrm{~m}^{\wedge} 2$ body mounted solar array around light tube, used for station keeping during spacecraft exchange

-Batteries sized for 0.5 hour attitude control contingency

- No active mirror thermal control during spacecraft exchange 


\section{Spacecraft Astrionics \& Power Systems}

6m Telescope - Spacecraft Astrionics and Power Systems

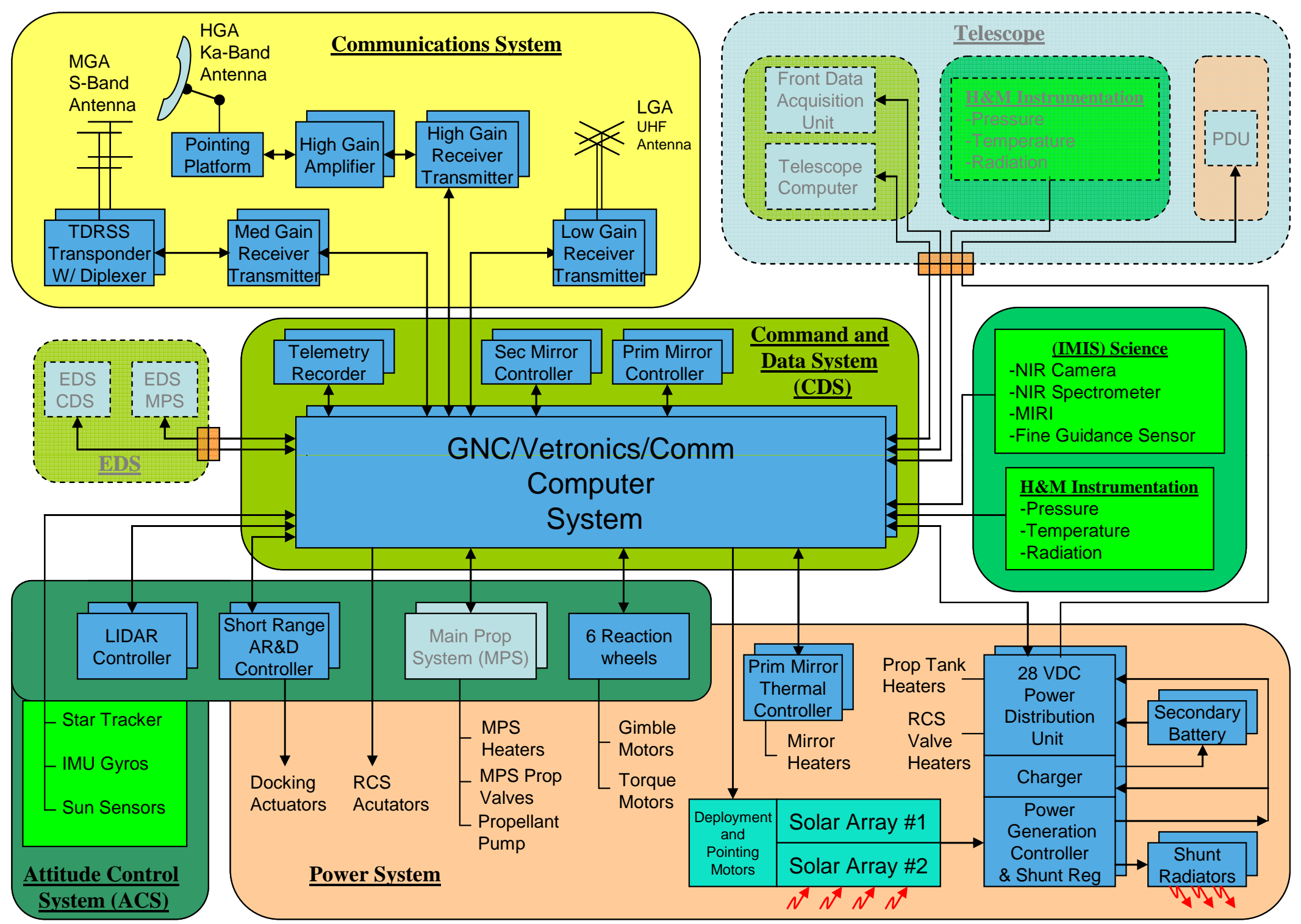




\section{Telescope Astrionics \& Power Systems}

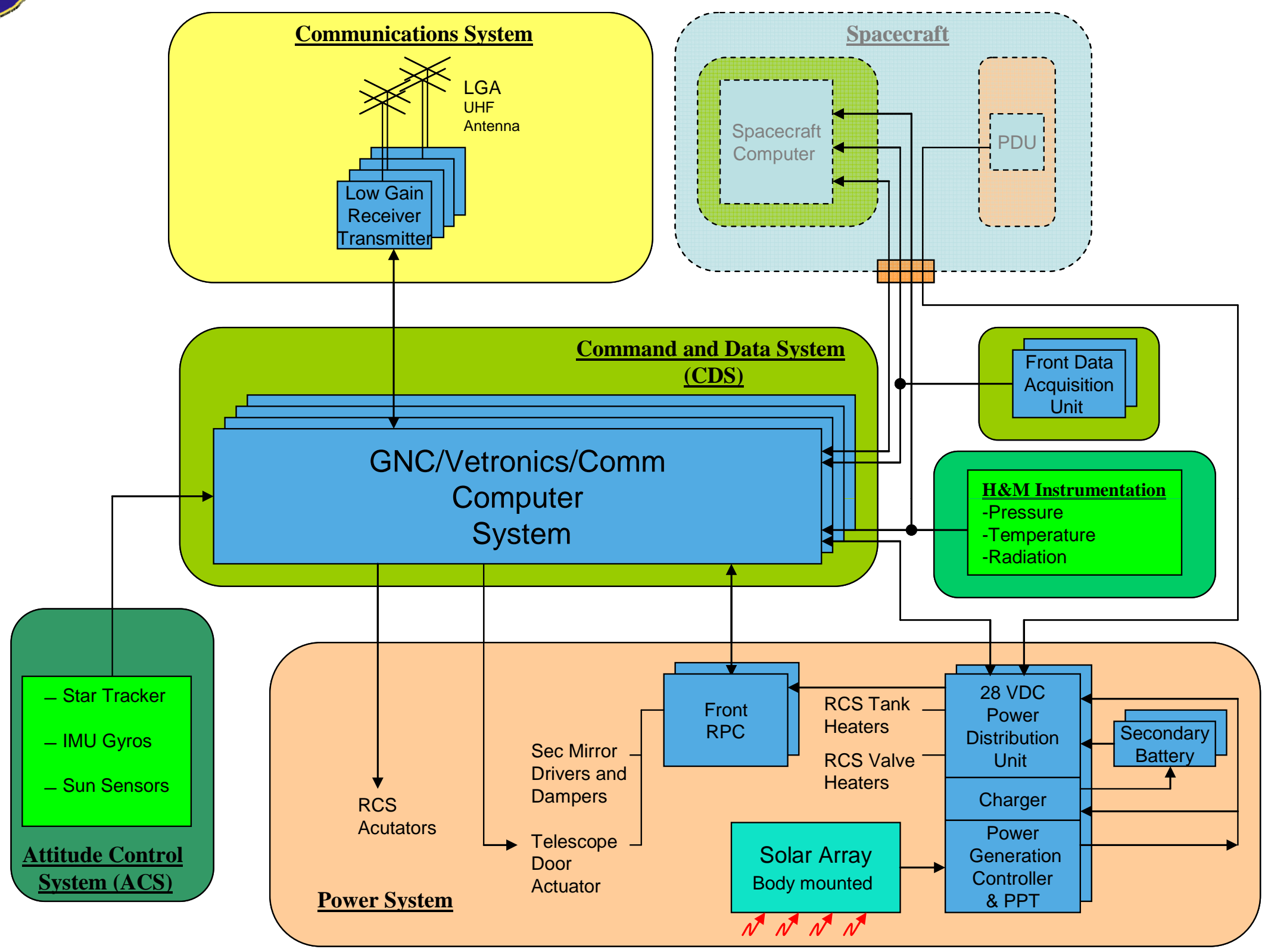




\section{Mission Life}

Initial Mission designed for a $5 \mathrm{yr}$ mission life (10 yr goal) should produce compelling science results well worth the modest mission cost.

But, there is no reason why the mission should end after 5 or even 10 years.

Hubble has demonstrated the value of on-orbit servicing

The telescope itself could last 30 or even 50 years. 


\section{0 to $\mathbf{5 0}$ year Mission Life}

Copy Ground Observatory Model - L2 Virtual Mountain

Design the observatory to be serviceable

Telescope has no inherent life limits

Replace Science Instruments every 3-5 yrs (or even 10 yrs)

Replacement

Spacecraft in ELV

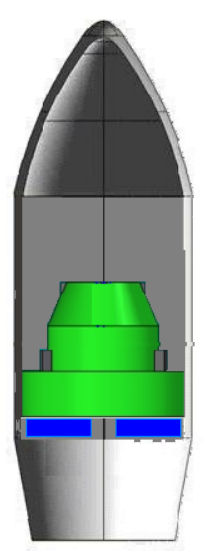

Autonomously docks to observatory; replaces all science instruments and ALL expendable components.

Spacecraft in

4.5 meter Payload Fairing
Observatory has split bus with on-board attitude control and propulsion during servicing. (already in mass budget) 


\section{AR\&D System Elements}

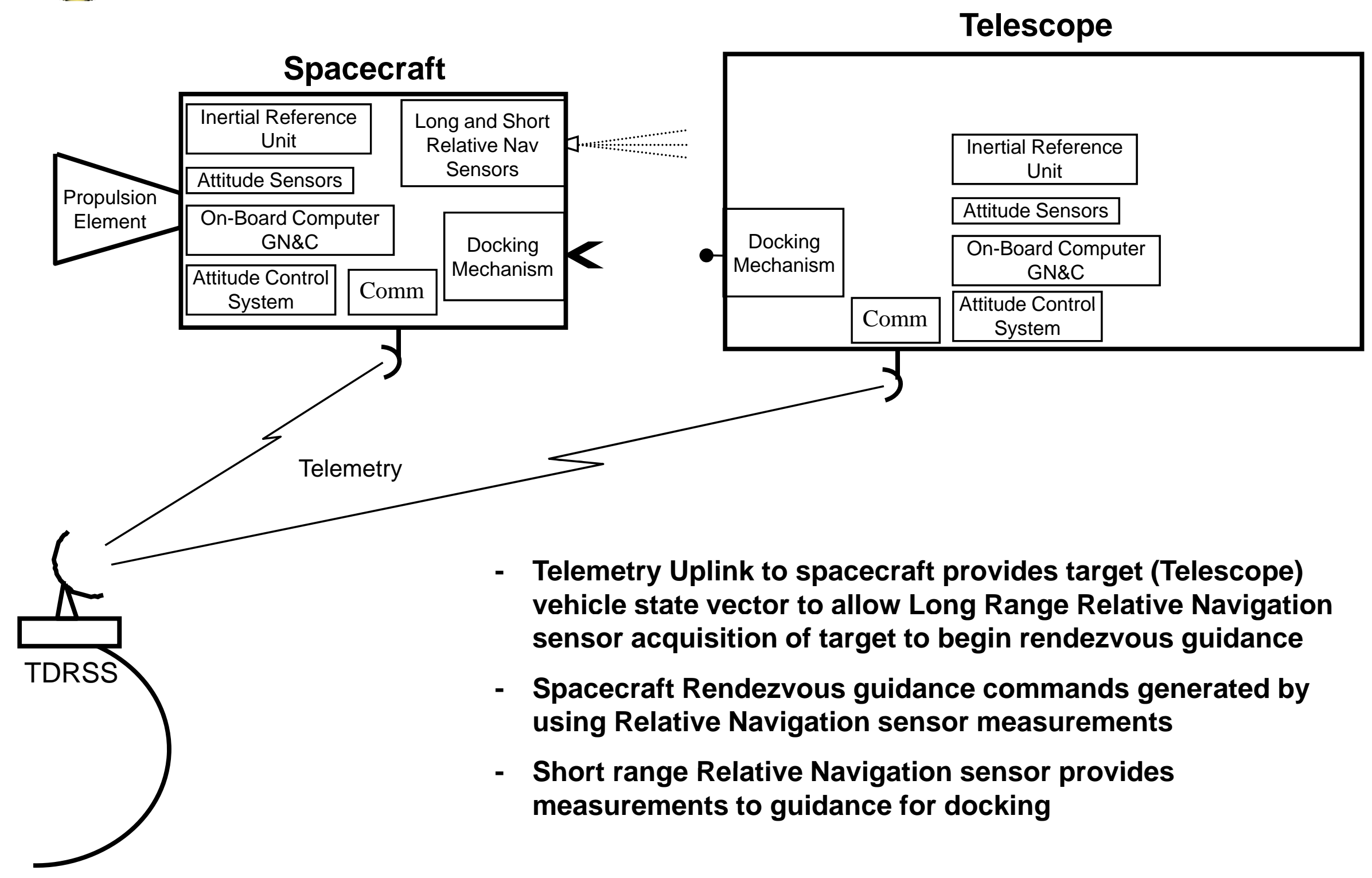




\section{Conclusions}

The unprecedented mass/volume capability of an Ares $\mathrm{V}$ enables the launch of 8 meter class monolithic space telescopes to the Earth-Sun L2 point.

NASA MSFC has determined that a 6 to 8 meter class telescope using a massive high-TRL ground observatory class monolithic primary mirror is feasible.

Mature, High-TRL design enables early deployment.

Science Instruments, Expendables and Limited Life Components can be replace periodically via Spacecraft Autonomous Rendezvous and Docking. 


\section{Any Question?}

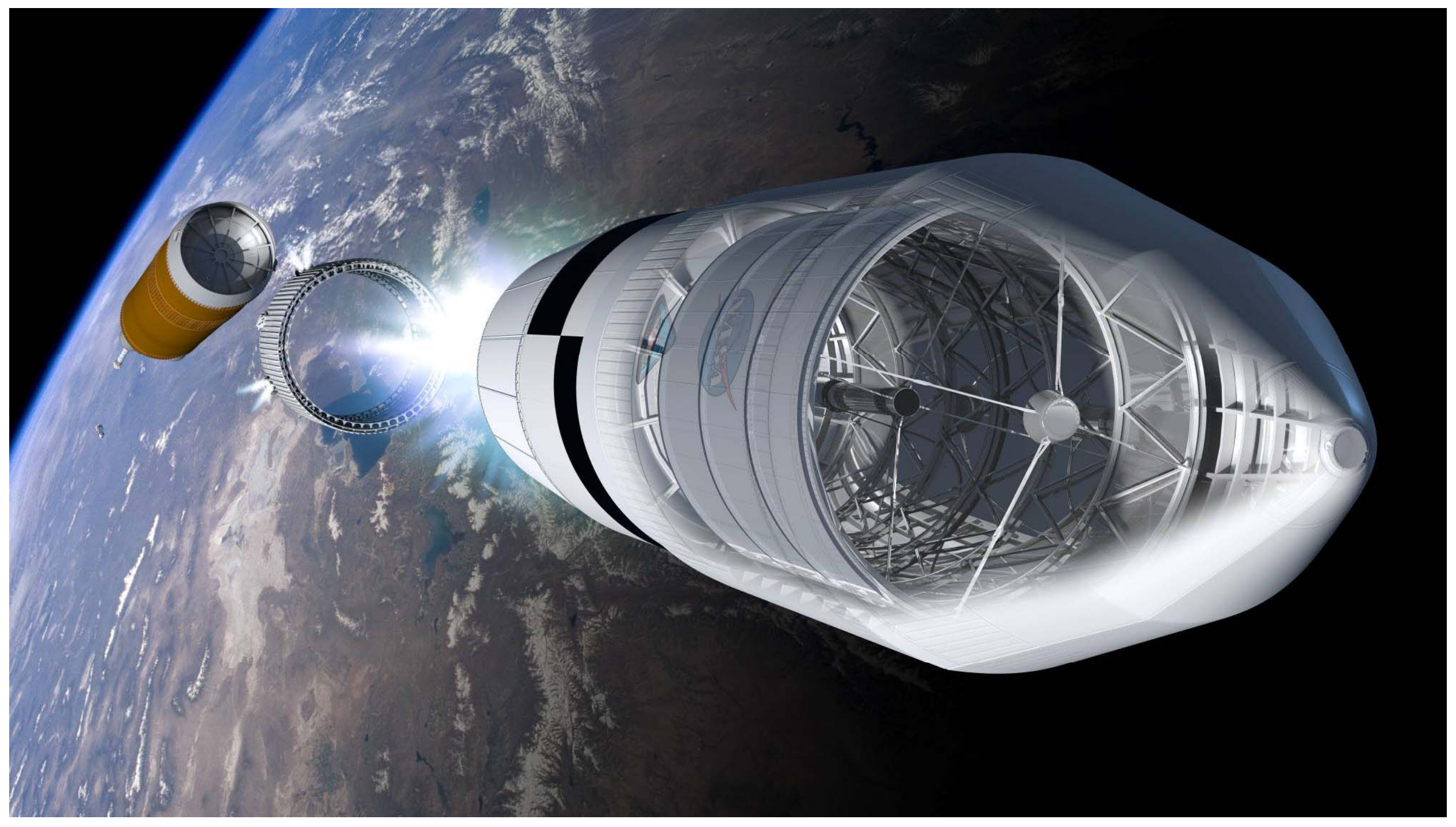

